Karl-Franzens Universität Graz

Technische Universität Graz

Medizinische Universität Graz

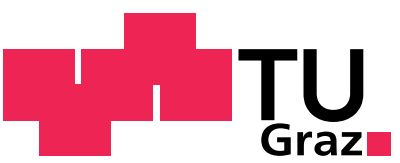

\title{
On Free Surface PDE Constrained Shape Optimization Problem
}

K. Kunisch H. Kasumba 
SFB sponsors:

- Austrian Science Fund (FWF)

- University of Graz

- Graz University of Technology

- Medical University of Graz

- Government of Styria

- City of Graz

Das Land

Steiermark

Stadt $\mathbf{G} \mathbf{R}$ A Z $\mathbf{Z}$ Wissenschaft 


\title{
On Free Surface PDE Constrained Shape Optimization Problem
}

\author{
H. Kasumba* and K. Kunisch ${ }^{\dagger}$
}

\begin{abstract}
Shape optimization problems in fluid dynamics governed by the free surface flows are considered. Such problems are inspired by the process of continuous casting of steel where optimization of large vortex structures in different metallurgical reactors is of paramount importance to ensure good quality output. The pseudo-transient approach is used as solution strategy for solving the free surface problem. Design sensitivities involving different cost functionals are derived using a formal Lagrangian framework. Numerical results are presented which indicate the success of the proposed algorithm for solving this free surface shape optimization problem.
\end{abstract}

Key words: Fluid dynamics, Free surface flow, Shape optimization.

\section{Introduction}

Many industrial processes contain a combination of fluid flow and free fluid surfaces. Examples of such processes are coating flows [15], thin film manufacturing processes [24], and continuous casting of steel [19]. The problem we consider in this paper is inspired by the latter process. Given the increasing demands related to quality of steel products, optimization of processes involved in continuous casting is required, e.g., process improvements in metallurgical reactors like ladle, tundish and mould. For this purpose, it is important to analyze the fluid flow and mixing that result from various processing parameters. Large vortex structures that appear in different metallurgical reactors influence the flotation and separation of non-metallic inclusions and therefore, the steel quality. These large vortex structures are partly due to the shape of metallurgical reactors. Different shapes produce different kinds of flows. Therefore, the optimal vortex control by means of the shape of these metallurgical reactors is important to ensure a good quality output. As an example, we consider the spout of a pouring tube reaching into a mold of liquid metal (see Figure 1). The control objective consists in determining its shape in such a way that the fluid remains as laminar as possible during the inflow. The location of the top surface $\Gamma_{2} \cup \Gamma_{7}$ is not known a priori and its determination is part of the problem. Therefore, in this work, we deal with a complicated problem namely, the control of the shape of a flow domain $\Omega$ with a free surface. This problem is an optimal control problem, where the free surface problem represents the state constraint and the parameters defining the subset of the boundary $\Gamma_{\mathrm{opt}}$ (to be specified later ) are the control. Such an optimization problem has been treated for the Bernoulli problem in [22] and apparently has not been considered previously for free

\footnotetext{
*Johann Radon Institute for Computational and Applied Mathematics, Austrian Academy of Sciences, Altenbergerstraße 69, A-4040 Linz, Austria, email: henry.kasumba@oeaw.at.

${ }^{\dagger}$ Institut für Mathematik und Wissenschaftliches Rechnen, Karl-Franzens-Universität Graz, Heinrichstraße 36, A-8010 Graz, Austria; email: karl.kunisch@uni-graz.at.
} 


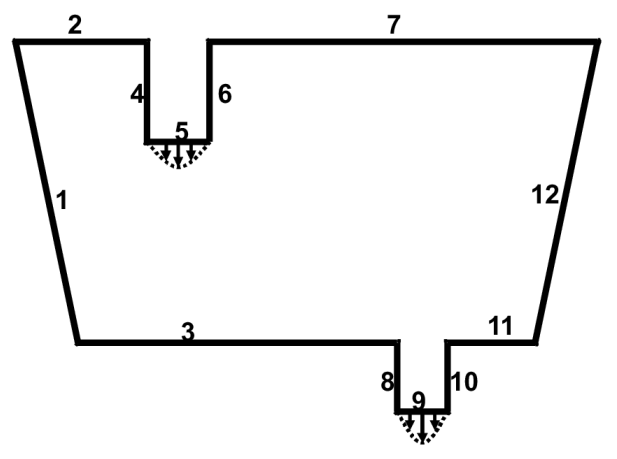

Figure 1: Model domain

surface flows governed by the Navier-stokes equations. Furthermore, in [22], a sensitivity analysis was performed for a discretized Bernoulli problem using an automatic differentiation technique. In the present work, we carry out the sensitivity analysis on the continuous formulation of the free surface problem. A possible way to realize numerically this optimization problem is to discard one of the boundary conditions on the free surface and to append it to the cost functional by using a penalty or augmented Lagrangian approach. Using this strategy, the state relation now becomes a classical non-linear boundary value problem with well-posed boundary data. Unfortunately, as noted in [22], this approach leads to serious convergence problems. A further disadvantage that was noted in [22] is that, depending on the formulation, a locally optimal triplet $\left(\Gamma_{\mathrm{opt}},\left(\mathbf{u}^{*}, p^{*}\right), \Omega^{*}\right)$ might not represent a physical solution to the free surface problem. For this reason, we solve the optimization problem in its original setting, i.e., we find a solution to the state equation first and then proceed to the upper level represented by the minimization of the cost functional. Since the free surface problem has to be solved several times for varying interfaces, one needs an efficient and robust solver for this type of problem. Possible solution strategies include trial methods, linearization methods (continuous or discrete) [4], and shape optimization methods [9]. Here we use the pseudo-transient approach, which is a trial method. The main advantage of this approach is that there is no explicit parametrization of the shape of the free boundary using, e.g., splines, but instead the boundary nodes can move freely. Moreover, this method converges linearly [4] (equivalent to the rate of convergence of a shape optimization method, which requires calculation of shape derivatives which is really only feasible for fairly simple systems).

The remainder of this paper is organized as follows. Section 2 describes the setting of the state and optimization problems. The sensitivity analysis of the optimization problems is given in Section 3. In Section 4, the numerical algorithm used to realize the optimization problems is given. Numerical examples that support the theoretical results are presented and the conclusions of this work are drawn. 


\section{Setting of the problem}

In this section the mathematical notations, the governing equations, and the corresponding boundary conditions of viscous fluid flows are presented. The equations are formulated in primitive variables, namely: the velocity components and the pressure.

\subsection{Notation}

Here we collect some notations and definitions that we need in our subsequent discussion. Throughout the paper, we restrict ourselves to the two dimensional case. Vectorvalued functions are indicated by bold letters. An element in $\mathbf{x} \in \mathbb{R}^{2}$, is denoted by $\mathbf{x}=\left(x_{1}, x_{2}\right)^{T}$ with norm $|\mathbf{x}|_{\mathbb{R}^{2}}=\left(\sum_{j=1}^{2} x_{j}^{2}\right)^{1 / 2}$. Two notations for the inner product in $\mathbb{R}^{2}$ shall be used, namely $(x, y)$ and $x \cdot y$, respectively. The latter shall be used in case of nested inner products. For a vector valued function $\mathbf{u}$, the gradient of $\mathbf{u}$, denoted by $\nabla \mathbf{u}$, is a second order tensor defined as $\nabla \mathbf{u}=[\nabla \mathbf{u}]_{i j}:=\left(\frac{\partial u_{j}}{\partial x_{i}}\right)_{i, j=1,2}$, where $[\nabla \mathbf{u}]_{i j}$ is the entry at the intersection of the $i^{\text {th }}$ row and $j^{\text {th }}$ column, while the Jacobian of $\mathbf{u}$, denoted by $D \mathbf{u}$, is the transpose of the gradient. The curl of a vector field $\mathbf{u}=\left(u_{1}, u_{2}\right)^{T} \in \mathbb{R}^{2}$, denoted by curl $\mathbf{u}$, is defined as curl $\mathbf{u}:=\frac{\partial u_{2}}{\partial x_{1}}-\frac{\partial u_{1}}{\partial x_{2}}$, while the curl of a scalar field $u$, denoted by curl $u$, is defined as curl $u:=\left(\frac{\partial u}{\partial x_{2}},-\frac{\partial u}{\partial x_{1}}\right)$. The determinant of the velocity gradient tensor of a vector field $\mathbf{u}=\left(u_{1}, u_{2}\right)^{T} \in \mathbb{R}^{2}$, denoted by det $\nabla \mathbf{u}(\mathbf{x})$, is defined as det $\nabla \mathbf{u}(\mathbf{x}):=\frac{\partial u_{1}}{\partial x_{1}} \frac{\partial u_{2}}{\partial x_{2}}-\frac{\partial u_{2}}{\partial x_{1}} \frac{\partial u_{1}}{\partial x_{2}}$. Furthermore, we define the tensor scalar product denoted by $\nabla \mathbf{u}: \nabla \boldsymbol{\psi}$ as

$$
\nabla \mathbf{u}: \nabla \boldsymbol{\psi}:=\left(\sum_{i, j=1}^{d} \frac{\partial u_{j}}{\partial x_{i}} \frac{\partial \psi_{j}}{\partial x_{i}}\right) \in \mathbb{R} .
$$

The unit outward normal and tangential vectors to the boundary $\partial \Omega$ shall be denoted by $\mathbf{n}=\left(n_{x_{1}}, n_{x_{2}}\right)$ and $\boldsymbol{\tau}=\left(-n_{x_{2}}, n_{x_{1}}\right)$, respectively. We denote by $H^{m}(\mathscr{S}), m \in \mathbb{R}^{+}$, the standard Sobolev space of order $m$ defined as

$$
H^{m}(\mathscr{S}):=\left\{u \in L^{2}(\mathscr{S}) \mid D^{\alpha} u \in L^{2}(\mathscr{S}), \text { for } 0 \leq|\alpha| \leq m\right\},
$$

where $D^{\alpha}$ is the weak (or distributional) partial derivative, and $\alpha$ is a multi-index. Here $\mathscr{S}$, is either the flow domain $\Omega$, or its boundary $\Gamma$, or part of its boundary. The norm $\|\cdot\|_{H^{m}(\mathscr{S})}$ associated with $H^{m}(\mathscr{S})$ is given by

$$
\|u\|_{H^{m}(\mathscr{S})}^{2}=\sum_{|\alpha| \leq m} \int_{\mathscr{S}}\left|D^{\alpha} u\right|^{2} d \mathbf{x} .
$$

Note that $H^{0}(\mathscr{S})=L^{2}(\mathscr{S})$ and $\|\cdot\|_{H^{0}(\mathscr{S})}=\|\cdot\|_{L^{2}(\mathscr{S})}$. For vector valued functions, we define the Sobolev space $\mathbf{H}^{m}(\mathscr{S})$ as

$$
\mathbf{H}^{m}(\mathscr{S}):=\left\{\mathbf{u}=\left(u_{1}, u_{2}\right) \mid u_{i} \in H^{m}(\mathscr{S}), \text { for } i=1,2\right\},
$$

and its associated norm

$$
\|\mathbf{u}\|_{\mathbf{H}^{m}(\mathscr{S})}^{2}=\sum_{i=1}^{2}\left\|u_{i}\right\|_{H^{m}(\mathscr{S})}^{2} .
$$




\subsection{State problem}

Let $\Omega$ be a connected bounded domain in $\mathbb{R}^{2}$ with a sufficiently piecewise regular boundary $\partial \Omega=\Gamma$. Suppose that an incompressible viscous flow occupies $\Omega$, and that the state equation for the flow is given by the following system of Navier-Stokes equations in non-dimensional form:

$$
\left\{\begin{aligned}
-v \Delta \mathbf{u}+(\mathbf{u} \cdot \nabla) \mathbf{u}+\nabla p & =\mathbf{f} \text { in } \Omega, \\
\operatorname{div} \mathbf{u}=0 & \text { in } \Omega .
\end{aligned}\right.
$$

Here $\mathbf{u}=\left(u_{1}, u_{2}\right)^{T}$ is the velocity field, $p$ the pressure, $v$ the kinematic viscosity of the fluid ( $v=\frac{1}{R e}>0$, where $R e$ is the Reynolds number) and $\mathbf{f}$ the density of external forces. The non-linear term $(\mathbf{u} \cdot \nabla) \mathbf{u}$ in (1) is a symbolic notation for the vector $\left(u_{1} \frac{\partial u_{1}}{\partial x_{1}}+u_{2} \frac{\partial u_{1}}{\partial x_{2}}, u_{1} \frac{\partial u_{2}}{\partial x_{1}}+u_{2} \frac{\partial u_{2}}{\partial x_{2}}\right)^{T}$. In order to make (1) well-posed, we have to impose appropriate boundary conditions. The boundaries $\Gamma_{5}, \Gamma_{9}$ and $\Gamma_{f} \equiv \Gamma_{2} \cup \Gamma_{7}$ in Figure 1 are the inflow, the outflow, and the free boundary, respectively. On each of the boundaries $\Gamma_{i}, i=1, \cdots, 12$, except $\Gamma_{f}$, two boundary conditions are imposed, i.e.,

$$
\left\{\begin{aligned}
\mathbf{u}=\mathbf{g} & =\left(0, u_{n}\right), \quad \text { on inflow } \Gamma_{5} \\
-p+v \frac{\partial \mathbf{u}}{\partial \mathbf{n}} \cdot \mathbf{n} & =0, \quad v \frac{\partial \mathbf{u}}{\partial \mathbf{n}} \cdot \mathbf{t}=0, \text { on outflow } \Gamma_{9} \\
\mathbf{u} \cdot \mathbf{n} & =0, \quad v \frac{\partial \mathbf{u}}{\partial \mathbf{n}} \cdot \mathbf{t}=0, \quad \text { on other fixed boundaries } \Gamma_{*}
\end{aligned}\right.
$$

where $\Gamma_{*}:=\Gamma_{1} \cup \Gamma_{3} \cup \Gamma_{8} \cup \Gamma_{10} \cup \Gamma_{11} \cup \Gamma_{12} \cup \Gamma_{6} \cup \Gamma_{4}$. Here the vector $\mathbf{g}$ is a given velocity at the inflow $\Gamma_{5}$. At the outflow $\Gamma_{9}$, a natural "do nothing" boundary condition proposed in [11] is imposed. Along other boundaries $\Gamma_{*}$, we require that the tangential stress acting on the fluid along these boundaries vanishes so that it slips freely along them in the tangential direction. In fact, if we are to impose the no-slip boundary conditions on $\Gamma_{4}$ and $\Gamma_{6}$, then a stress singularity would result at contact points where the boundaries $\Gamma_{2}$ and $\Gamma_{7}$, meet the boundaries $\Gamma_{4}$ and $\Gamma_{6}$, respectively [16]. The same reasoning holds at intersection points where $\Gamma_{2}$ and $\Gamma_{7}$, meet the boundaries $\Gamma_{1}$ and $\Gamma_{12}$, respectively. Therefore, the choice of the free-slip boundary condition (2.3) on $\Gamma_{*}$ suggests itself.

If we assume zero ambient pressure and negligible surface tension effects, then the 3 boundary conditions on the free surface corresponding to the kinematic, normal and tangential stress balances can be expressed as

$$
\begin{aligned}
\mathbf{u} \cdot \mathbf{n} & =0, \quad \text { on } \Gamma_{f}, \\
-p+v \frac{\partial \mathbf{u}}{\partial \mathbf{n}} \cdot \mathbf{n} & =0, \quad \text { and } v \frac{\partial \mathbf{u}}{\partial \mathbf{n}} \cdot \mathbf{t}=0 \text { on } \Gamma_{f}
\end{aligned}
$$

respectively [7]. 


\subsection{Pseudo-transient approach for the state problem}

We can only apply two of the 3 boundary conditions in (3a-3b). The third is then used to solve for the unknown free surface position. Two schemes exist for solving the state problem (1-3) using a Picard type technique, namely the kinematic and normal stress update schemes. The former is stable for flows where surface tension effects are insignificant [23]. Therefore, we use this update scheme in this work. We solve the boundary value problem (1) together with boundary conditions (2) and (3b) on an initial domain $\Omega_{0}$. The solution $(\mathbf{u}, p)$ on $\Omega_{0}$ will not satisfy the boundary condition (3a). Hence, $\Gamma_{f}$ needs to be shifted iteratively in order to find a domain $\Omega$ satisfying it. For this purpose, a pseudo time $t$ is introduced and we assume that the instantaneous normal velocity of the free surface is equal to the normal component of the fluid velocity at the free surface at all times. This consequently translates (3a) to

$$
\mathbf{n} \cdot\left(\frac{d \mathbf{x}}{d t}-\mathbf{u}\right)=0 \text { on } \Gamma_{f},
$$

where $\mathbf{x}$ is the vector representing the free surface. For a converged solution of the free surface, (4) reduces to (3a). The algorithmic steps can now be summarized as follows.

Algorithm 1 Free surface flow solution algorithm

1. Set the initial grid $\Omega_{0}$ and the free surface position $\Gamma_{0}$.

2. Solve the Navier-Stokes equations (1), (2) and (3b) to compute the velocity field $\mathbf{u}^{k}=\left(u_{1}^{k}, u_{2}^{k}\right)^{T}$ and pressure $p^{k}$ on this geometry.

3. Compute the new geometry $\left(\Omega_{k+1}\right)$ by updating the coordinates at the free surface with aid of (4) using $\left(u_{1}^{k}, u_{2}^{k}\right)^{T}$ and regenerating the mesh such that

$$
\left(x_{1}^{k+1}, x_{2}^{k+1}\right)^{T}=\left(x_{1}^{k}, x_{2}^{k}\right)^{T}+\Delta t\left(\mathbf{u}^{k} \cdot \mathbf{n}\right)\left(n_{x_{1}}, n_{x_{2}}\right)^{T} \text { where } \Delta t \text { is the step size }
$$

4. If free surface is moving go to 2 .

Remark 2.1. Note that the coordinates $\left(x_{1}, x_{2}\right)^{T}$ in step 3 of Algorithm 1 are defined locally on the free surface. Thus, we need to provide for the interior node movements to avoid mesh distortions. For this purpose, we solve the following Poisson problem to obtain the interior and boundary vertex displacements,

$$
\begin{aligned}
\Delta \boldsymbol{\Theta} & =0, \text { in } \Omega, \boldsymbol{\Theta} \cdot \mathbf{n}=0, \text { and }\left(\frac{\partial \boldsymbol{\Theta}}{\partial \mathbf{n}}\right) \cdot \mathbf{t}=0, \text { on } \Gamma_{S}, \\
\boldsymbol{\Theta} & =0, \text { on } \Gamma_{N S}, \quad \boldsymbol{\Theta}=(\mathbf{u} \cdot \mathbf{n}) \mathbf{n}, \text { on } \Gamma_{f},
\end{aligned}
$$

where $\Gamma_{S}:=\Gamma_{1} \cup \Gamma_{4} \cup \Gamma_{6} \cup \Gamma_{12}$ and $\Gamma_{N S}:=\Gamma_{3}, \Gamma_{5}, \Gamma_{8}, \Gamma_{9}, \Gamma_{10}, \Gamma_{11}$. The choice of the boundary conditions on $\Gamma_{S} \cup \Gamma_{N S} \cup \Gamma_{f}$ is dictated by the physics of the problem. For instance on $\Gamma_{S}$, a slip boundary condition is chosen since we want the mesh nodes to move freely along the tangential direction. Mesh nodes on $\Gamma_{N S}$ are fixed, while on $\Gamma_{f}$, 
they move with the normal component of the fluid velocity, in the direction of the unit outward normal vector to $\Gamma_{f}$. The mesh update at the $k^{\text {th }}$ time step is now given by

$$
\left(x_{1}^{k+1}, x_{2}^{k+1}\right)^{T}=\left(x_{1}^{k}, x_{2}^{k}\right)^{T}+\Delta t \boldsymbol{\Theta}^{k},
$$

where $\left(x_{1}, x_{2}\right)$ in (7) are global node locations. Since we are using the forward Euler time stepping algorithm, we must use a small step size $\Delta t$ to ensure stability of the algorithm [23].

In order to test Algorithm 1, the following flow parameters are chosen, i.e., $\mathbf{f}=(-1,0)$, the inflow speed $\mathbf{g}=(-0.56,0)$, and $R e=50$. The computational domain in Figure 1 is discretized by triangular elements generated by the bi-dimensional anisotropic mesh generator [10]. The Navier-Stokes equations (1), (2) and (3b), are then discretized using the Galerkin finite-element method. We use polynomial of degree two for the velocity approximation and polynomial of degree one for the pressure. This results in a set of nonlinear algebraic equations that may be represented in matrix form as

$$
\mathbf{K}(\overline{\mathbf{u}}) \overline{\mathbf{u}}=\mathbf{F},
$$

where $\mathbf{K}$ is the global system matrix, $\overline{\mathbf{u}}$ is the global vector of unknowns (velocities and pressures), and $\mathbf{F}$ is a vector that includes the effects of body forces and boundary conditions. A fixed point iteration procedure, namely, a Picard iteration which may be written as

$$
\mathbf{K}\left(\overline{\mathbf{u}}_{\mathbf{i}}\right) \overline{\mathbf{u}}_{\mathbf{i}+\mathbf{1}}=\mathbf{F}
$$

is used to solve the discrete system (8). Here, the nonlinearity is evaluated at the known iterate $\overline{\mathbf{u}}_{\mathbf{i}}$, and a non-symmetric linear system is formed at each iteration. This linear system is solved by a multi-frontal Gauss LU factorization implemented in the package UMFPACK [5]. The flow field patterns in Figure 2 are obtained. In Figure 2(a), the

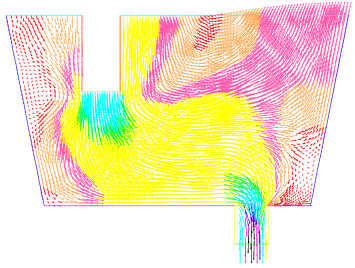

(a) iter $=2$

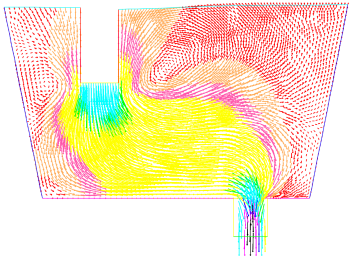

(b) iter $=6$

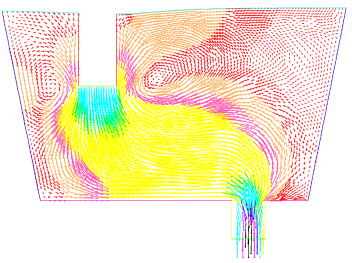

(c) iter $=17$

Figure 2: Snapshots of velocity field at different iteration numbers

velocity field lines point out of $\Gamma_{f}$ and therefore, the flow field does not satisfy $\mathbf{u} \cdot \mathbf{n}=0$ on $\Gamma_{f}$. Hence, we run Algorithm 1 until $\|\Theta\|_{L^{2}}<10^{-3}$ and we stop. This stopping criterion is met after 17 iterations. The corresponding geometry and flow solving the free surface problem are depicted in Figure 2 (c).

\subsection{Optimization problems}

Our objective is to find a domain that solves the free surface problem (1-3) and at the same time possesses "minimal" vortices within the containment. For this purpose, 
we utilize cost functionals for vortex reduction in fluid dynamics. These include the tracking-type functional

$$
J_{1}(\mathbf{u}, \Omega)=\frac{1}{2} \int_{\Omega}\left|\mathbf{u}(\mathbf{x})-\mathbf{u}_{d}(\mathbf{x})\right|^{2} d \mathbf{x}
$$

which matches a given flow field $\mathbf{u}$ to some given desired flow field $\mathbf{u}_{d}$ without vortices,

$$
J_{2}(\mathbf{u}, \Omega)=\frac{1}{2} \int_{\Omega}|\operatorname{curl} \mathbf{u}(\mathbf{x})|^{2} d \mathbf{x},
$$

which minimizes of the curl of the velocity field [1]. Furthermore, we utilize

$$
J_{3}(\mathbf{u}, \Omega)=\int_{\Omega} g_{3}(\operatorname{det} \nabla \mathbf{u}) d \mathbf{x}, \text { where } g_{3}(t)= \begin{cases}0 & t \leq 0 \\ \frac{t^{3}}{t^{2}+1} & t>0\end{cases}
$$

which was more recently introduced in ( see, e.g., [12] and references there in ) for $2 \mathrm{D}$ flows. It penalizes the complex eigenvalues of $\nabla \mathbf{u}$ which are responsible for the swirling motion in a given flow. The optimization problems can be formulated as follows: Find $\Omega$ over a class of admissible domains $\mathscr{U}_{a d}$ such that the functionals $J_{1}, J_{2}$ and $J_{3}$ are minimized subject to the Navier-Stokes equations (1-3). More precisely the optimization problems read

$$
\left\{\begin{array}{c}
\min _{\Omega \in \mathscr{U}_{a d}} J_{i}(\mathbf{u}, \Omega) \\
\text { subject to }(1-3) .
\end{array}\right.
$$

The set $\mathscr{U}_{a d}$ is set up in such a way that problem (12) possesses at least one solution $(\Omega, \mathbf{u}, p)$.

\section{Sensitivity analysis}

In this section we discuss the necessary optimality conditions for (12). We implicitly assume that the underlying functions are regular enough to ensure well posedness of all the operations. In order to set up the optimality system, a common technique is to introduce a family of perturbations $\left\{\Omega_{t}: t>0\right\}$ of a given admissible domain $\Omega$. The relationship of these perturbations to the set of admissible domains $\mathscr{U}_{\text {ad }}$ will be discussed in Section 4. This family of perturbations can be constructed for instance by perturbation of the identity, see, e.g., [21],[6],[9]. Let $D \subset \mathbb{R}^{2}$ be a fixed, open and bounded hold all domain with a piecewise $C^{1,1}$ boundary $\partial D$. Let $\Omega \subset \bar{D}$ be open and let

$$
\mathscr{F}=\left\{\mathbf{h} \in C^{1,1}(\bar{D}):\left.\mathbf{h}\right|_{\partial D}=0\right\}
$$

be the space of deformation fields which define for $t>0$, a perturbation of $\Omega$ by

$$
\begin{aligned}
T_{t}: D & \mapsto \mathbb{R}^{2}, \\
\mathbf{x} & \mapsto T_{t}(\mathbf{x})=\mathbf{x}+t \mathbf{h}(\mathbf{x}) .
\end{aligned}
$$


Then for each $\mathbf{h} \in \mathscr{F}$, there exists $\tau_{0}>0$ such that $T_{t}(D)=D$ and $\left\{T_{t}\right\}$ is a family of $C^{1,1}$-diffeomorphisms for $|t|<\tau_{0}$ [21]. For each $t \in \mathbb{R}$ with $|t|<\tau_{0}$, we set

$$
\Omega_{t}=T_{t}(\Omega), \Gamma_{t}=T_{t}(\Gamma) .
$$

Thus, $\Omega_{0}=\Omega, \Gamma_{0}=\Gamma, \Omega_{t} \subset D$. In what follows, we assume that $\mathbf{f}$ and $\mathbf{g}$ in (1) and (2.1), respectively, are fixed functions defined on $D$. The shape derivative of $J$ at $\Omega$ in the direction of the deformation field $\mathbf{h}$ is defined as the limit

$$
d J(\mathbf{u}, \Omega) \mathbf{h}=\lim _{t \rightarrow 0^{+}} \frac{J\left(\mathbf{u}_{t}, \Omega_{t}\right)-J(\mathbf{u}, \Omega)}{t},
$$

provided that it exists. Here $\mathbf{u}_{t}$ is the solution of (1-3) when we replace $\Omega$ by $\Omega_{t}$. If the mapping $\mathbf{h} \mapsto d J(\mathbf{u}, \Omega) \mathbf{h}$ is linear and continuous, then we say that $J(\mathbf{u}, \Omega)$ is shape differentiable [21]. If $\Omega$ is sufficiently smooth such that $d J(\mathbf{u}, \Omega) \mathbf{h}=\int_{\Gamma_{\mathrm{opt}}} \nabla j \mathbf{n} \cdot \mathbf{h} d s$, for some scalar-valued function $j$, then we call $\nabla j \mathbf{n}$ the shape gradient of $J(\mathbf{u}, \Omega)$. We shall now discuss in a formal manner how to obtain the shape derivative for $J_{i}(\mathbf{u}, \Omega), i=$ $1,2,3$.

\subsection{The formal Lagrangian approach}

In order to set up the optimality system for the optimization problem, we introduce the Lagrange functional $\mathscr{L}$ as follows:

$$
\begin{array}{r}
\mathscr{L}\left(\mathbf{u}, p, \mathbf{v}, q, \boldsymbol{\eta}, \boldsymbol{\gamma}, \mu_{1}, \mu_{2}, \Omega\right)=\int_{\Omega} j_{i}(\mathbf{u}) d \mathbf{x}-\int_{\Omega} \mathbf{v}(-\alpha \Delta \mathbf{u}+(\mathbf{u} \cdot \nabla) \mathbf{u}+\nabla p-\mathbf{f}) d \mathbf{x}+ \\
\int_{\Omega} q \operatorname{div} \mathbf{u} d \mathbf{x}-\int_{\Gamma_{5}}(\mathbf{u}-\mathbf{g}) \boldsymbol{\eta} d s-\int_{\Gamma_{9}}\left(-p \mathbf{n}+\alpha \frac{\partial \mathbf{u}}{\partial \mathbf{n}}\right) \boldsymbol{\gamma} d s-\int_{\Gamma_{*}}(\mathbf{u} \cdot \mathbf{n}) \mu_{1} d s \\
-\int_{\Gamma_{*}}\left(\alpha \frac{\partial \mathbf{u}}{\partial \mathbf{n}} \cdot \mathbf{t}\right) \mu_{2} d s,
\end{array}
$$

where $\mathbf{v}, q, \boldsymbol{\eta}, \boldsymbol{\gamma}, \mu_{1}$ and $\mu_{2}$ are Lagrange multipliers to enforce the momentum equation, the incompressibility condition, as well as the boundary conditions. Note that $\mu_{1}$ and $\mu_{2}$ are scalar valued Lagrange multipliers. The boundary conditions

$$
\left\{\begin{aligned}
-p \mathbf{n}+\alpha \frac{\partial \mathbf{u}}{\partial \mathbf{n}} & =0, \\
\mathbf{u} \cdot \mathbf{n} & =0, \text { on free surface } \Gamma_{f}=\Gamma_{2} \cup \Gamma_{7},
\end{aligned}\right.
$$

have not been enforced through the Lagrange functional. Therefore, any variations $\tilde{\mathbf{u}}$ and $\tilde{p}$ in the states $\mathbf{u}$ and $p$ must be such that

$$
\left\{\begin{aligned}
-\tilde{p} \mathbf{n}+\alpha \frac{\partial \tilde{\mathbf{u}}}{\partial \mathbf{n}} & =0, \text { on free surface } \Gamma_{f} \\
\tilde{\mathbf{u}} \cdot \mathbf{n} & =0, \text { on free surface } \Gamma_{f} .
\end{aligned}\right.
$$

To obtain the optimality system for the optimization problem (12), we take the first variation of $\mathscr{L}$ with respect to Lagrange multipliers $\left(\mathbf{v}, q, \boldsymbol{\eta}, \boldsymbol{\gamma}, \mu_{1}, \mu_{2}\right)$, state variables 
$(\mathbf{u}, p)$ and the domain $\Omega$ in directions $\left(\tilde{\mathbf{v}}, \tilde{q}, \tilde{\boldsymbol{\eta}}, \tilde{\boldsymbol{\gamma}}, \tilde{\mu}_{1}, \tilde{\mu}_{2}\right),(\tilde{\mathbf{u}}, \tilde{p})$ and $\mathbf{h}$, respectively, to zero, i.e.,

$$
\begin{aligned}
\mathscr{L}_{\mathbf{v}, q, \boldsymbol{\eta}, \boldsymbol{\gamma}, \mu_{1}, \mu_{2}}\left(\tilde{\mathbf{v}}, \tilde{q}, \tilde{\boldsymbol{\eta}}, \tilde{\boldsymbol{\gamma}}, \tilde{\mu}_{1}, \tilde{\mu}_{2}\right) & =0, \\
\mathscr{L}_{\mathbf{u}, p}(\tilde{\mathbf{u}}, \tilde{p}) & =0, \\
\mathscr{L}_{\Omega} \mathbf{h} & =0 .
\end{aligned}
$$

Equation (19) leads to the state problem (1-3), (20) to the adjoint problem that we derive in Theorem 3.1, and (21) leads to the optimality conditions, whose derivation is discussed in subsection 3.2.

Theorem 3.1. Formally the adjoint equations associated to (1-3) are given by

$$
\left\{\begin{array}{l}
-\alpha \Delta \mathbf{v}-(\mathbf{u} \cdot \nabla) \mathbf{v}+[\nabla \mathbf{u}] \cdot \mathbf{v}+\nabla q=j_{i}^{\prime}(\mathbf{u}), \text { in } \Omega, \\
\operatorname{div} \mathbf{v}=0, \text { in } \Omega, \\
\mathbf{v} \cdot \mathbf{n}=0, \mathbf{t} \cdot\left[q \mathbf{n}-(\mathbf{u} \cdot \mathbf{n}) \mathbf{v}-\alpha \frac{\partial \mathbf{v}}{\partial \mathbf{n}}\right]=0, \text { on } \Gamma_{*}, \\
\mathbf{v}=0, \text { on } \Gamma_{5}, \\
\mathbf{v} \cdot \mathbf{n}=0, \quad\left[q \mathbf{n}-(\mathbf{u} \cdot \mathbf{n}) \mathbf{v}-\alpha \frac{\partial \mathbf{v}}{\partial \mathbf{n}}\right]=0, \text { on } \Gamma_{f}, \\
q \mathbf{n}-(\mathbf{u} \cdot \mathbf{n}) \mathbf{v}-\alpha \frac{\partial \mathbf{v}}{\partial \mathbf{n}}=0, \text { on } \Gamma_{9} .
\end{array}\right.
$$

Proof. (a) Setting the first variation of $\mathscr{L}$ with respect to $p$ in direction $\tilde{p}$ to zero, i.e., $\mathscr{L}_{p} \tilde{p}=0$, leads to

$$
\mathscr{L}_{p} \tilde{p}=-\int_{\Omega} \mathbf{v} \nabla \tilde{p} d \mathbf{x}-\int_{\Gamma_{9}}(-\boldsymbol{\gamma} \cdot \mathbf{n}) \tilde{p} d s=0 .
$$

Integrating by parts to remove where possible, derivatives from $\tilde{p}$, one obtains

$$
\int_{\Omega} \operatorname{div} \mathbf{v} \tilde{p} d \mathbf{x}-\int_{\Gamma}(\mathbf{v} \cdot \mathbf{n}) \tilde{p} d s-\int_{\Gamma_{9}}(-\boldsymbol{\gamma} \cdot \mathbf{n}) \tilde{p} d s=0 .
$$

Choosing $\tilde{p} \in C_{0}^{\infty}(\Omega)$, we obtain

$$
\int_{\Omega} \operatorname{div} \mathbf{v} \tilde{p} d \mathbf{x}=0
$$

Since $C_{0}^{\infty}(\Omega)$ is dense in $L^{2}(\Omega)$, the relation (23) holds almost every where in $\Omega$. Hence,

$$
\operatorname{div} \mathbf{v}=0, \text { in } \Omega .
$$

By similar arguments, we can show that

$$
\begin{aligned}
& \mathbf{v} \cdot \mathbf{n}=0 \text { on } \Gamma_{*} \cup \Gamma_{5} \cup \Gamma_{2} \cup \Gamma_{7}, \\
& \boldsymbol{\gamma} \cdot \mathbf{n}=\mathbf{v} \cdot \mathbf{n} \text { on } \Gamma_{9} .
\end{aligned}
$$


(b) Setting the variation of $\mathscr{L}$ with respect to the state $\mathbf{u}$ in the direction $\tilde{\mathbf{u}}$ equal to zero results in

$$
\begin{aligned}
\mathscr{L}_{\mathbf{u}} \tilde{\mathbf{u}} & =\int_{\Omega} j_{i}^{\prime}(\mathbf{u}) \tilde{\mathbf{u}} d \mathbf{x}-\int_{\Omega} \mathbf{v} \cdot(-\alpha \Delta \tilde{\mathbf{u}}+(\tilde{\mathbf{u}} \cdot \nabla) \mathbf{u}+(\mathbf{u} \cdot \nabla) \tilde{\mathbf{u}}) d \mathbf{x}+\int_{\Omega} q \operatorname{div} \tilde{\mathbf{u}} d \mathbf{x} \\
& -\int_{\Gamma_{5}} \tilde{\mathbf{u}} \cdot \boldsymbol{\eta} d s-\int_{\Gamma_{9}} \alpha \frac{\partial \tilde{\mathbf{u}}}{\partial \mathbf{n}} \cdot \boldsymbol{\gamma} d s-\int_{\Gamma_{*}}(\tilde{\mathbf{u}} \cdot \mathbf{n}) \mu_{1} d s-\int_{\Gamma_{*}}\left(\alpha \frac{\partial \tilde{\mathbf{u}}}{\partial \mathbf{n}} \cdot \mathbf{t}\right) \mu_{2} d s=0,
\end{aligned}
$$

where $\tilde{\mathbf{u}}$ denotes an arbitrary variation in the state variable $\mathbf{u}$ satisfying (18). Integrating by parts to remove where possible, derivatives from $\tilde{\mathbf{u}}$, one obtains

$$
\begin{array}{r}
\int_{\Omega}\left(j_{i}^{\prime}(\mathbf{u})+\alpha \Delta \mathbf{v}+(\mathbf{u} \cdot \nabla) \mathbf{v}-[\nabla \mathbf{u}] \cdot \mathbf{v}-\nabla q\right) \tilde{\mathbf{u}} d \mathbf{x}+\int_{\Gamma}\left(\alpha \frac{\partial \tilde{\mathbf{u}}}{\partial \mathbf{n}} \cdot \mathbf{v}-\alpha \frac{\partial \mathbf{v}}{\partial \mathbf{n}} \cdot \tilde{\mathbf{u}}\right) d s \\
+\int_{\Gamma}[\tilde{\mathbf{u}} \cdot(q \mathbf{n})-(\mathbf{u} \cdot \mathbf{n})(\mathbf{v} \cdot \tilde{\mathbf{u}})] d s-\int_{\Gamma_{5}} \tilde{\mathbf{u}} \cdot \boldsymbol{\eta} d s-\int_{\Gamma_{9}} \alpha \frac{\partial \tilde{\mathbf{u}}}{\partial \mathbf{n}} \cdot \boldsymbol{\gamma} d s-\int_{\Gamma_{*}}(\tilde{\mathbf{u}} \cdot \mathbf{n}) \mu_{1} d s \\
-\int_{\Gamma_{*}}\left(\alpha \frac{\partial \tilde{\mathbf{u}}}{\partial \mathbf{n}} \cdot \mathbf{t}\right) \mu_{2} d s=0 .
\end{array}
$$

(i) Choosing $\tilde{\mathbf{u}} \in C_{0}^{\infty}(\Omega)^{2}$, and using density arguments as before leads to

$$
-\alpha \Delta \mathbf{v}-(\mathbf{u} \cdot \nabla) \mathbf{v}+[\nabla \mathbf{u}] \cdot \mathbf{v}+\nabla q=j_{i}^{\prime}(\mathbf{u}), \text { in } \Omega .
$$

Thus, we remain with the expression,

$$
\begin{aligned}
& \int_{\Gamma}\left(\alpha \frac{\partial \tilde{\mathbf{u}}}{\partial \mathbf{n}} \cdot \mathbf{v}-\alpha \frac{\partial \mathbf{v}}{\partial \mathbf{n}} \cdot \tilde{\mathbf{u}}\right) d s+\int_{\Gamma}[q(\tilde{\mathbf{u}} \cdot \mathbf{n})-(\mathbf{u} \cdot \mathbf{n})(\mathbf{v} \cdot \tilde{\mathbf{u}})] d s-\int_{\Gamma_{5}} \tilde{\mathbf{u}} \cdot \boldsymbol{\eta} d s \\
& -\int_{\Gamma_{9}} \alpha \frac{\partial \tilde{\mathbf{u}}}{\partial \mathbf{n}} \cdot \boldsymbol{\gamma} d s-\int_{\Gamma_{*}}(\tilde{\mathbf{u}} \cdot \mathbf{n}) \mu_{1} d s-\int_{\Gamma_{*}}\left(\alpha \frac{\partial \tilde{\mathbf{u}}}{\partial \mathbf{n}} \cdot \mathbf{t}\right) \mu_{2} d s=0 .
\end{aligned}
$$

(ii) Choosing $\tilde{\tilde{\mathbf{u}}} \in C^{\infty}(\Gamma)^{2}$, we can find an extension $\tilde{\mathbf{u}} \in C^{\infty}(\Omega)^{2}$ such that $\left.\tilde{\mathbf{u}}\right|_{\Gamma}=\tilde{\tilde{\mathbf{u}}}$ and $\left.\tilde{\mathbf{u}}\right|_{\Gamma \backslash \Gamma_{5}}=0$. Consequently, we have that

$$
\int_{\Gamma_{5}}\left(\alpha \frac{\partial \tilde{\tilde{\mathbf{u}}}}{\partial \mathbf{n}} \cdot \mathbf{v}-\alpha \frac{\partial \mathbf{v}}{\partial \mathbf{n}} \cdot \tilde{\tilde{\mathbf{u}}}\right) d s+\int_{\Gamma_{5}} q(\tilde{\tilde{\mathbf{u}}} \cdot \mathbf{n})-(\mathbf{u} \cdot \mathbf{n})(\mathbf{v} \cdot \tilde{\tilde{\mathbf{u}}})-\tilde{\tilde{\mathbf{u}}} \cdot \boldsymbol{\eta} d s=0
$$

from which we obtain

$$
\mathbf{v}=0, \text { and } \boldsymbol{\eta}=q \mathbf{n}-(\mathbf{u} \cdot \mathbf{n}) \mathbf{v}-\alpha \frac{\partial \mathbf{v}}{\partial \mathbf{n}}, \text { on } \Gamma_{5} .
$$

(iii) Similarly choosing $\tilde{\tilde{\mathbf{u}}} \in C^{\infty}(\Gamma)^{2}$, we can find an extension $\tilde{\mathbf{u}} \in C^{\infty}(\Omega)^{2}$ such that $\left.\tilde{\mathbf{u}}\right|_{\Gamma}=\tilde{\tilde{\mathbf{u}}}$ and $\left.\tilde{\mathbf{u}}\right|_{\Gamma \backslash \Gamma_{*}}=0$. This leads to

$$
\int_{\Gamma_{*}}\left(\alpha \frac{\partial \tilde{\mathbf{u}}}{\partial \mathbf{n}} \cdot \mathbf{v}+\left[q \mathbf{n}-(\mathbf{u} \cdot \mathbf{n}) \mathbf{v}-\alpha \frac{\partial \mathbf{v}}{\partial \mathbf{n}}\right] \cdot \tilde{\mathbf{u}}-\left(\alpha \frac{\partial \tilde{\mathbf{u}}}{\partial \mathbf{n}} \cdot \mathbf{t}\right) \mu_{2}-(\tilde{\mathbf{u}} \cdot \mathbf{n}) \mu_{1}\right) d s=0 .
$$


Re-writing the first and second terms in (26) in component-wise form, we obtain using that $\tilde{\mathbf{v}}=(\tilde{\mathbf{v}} \cdot \mathbf{n}) \mathbf{n}+(\tilde{\mathbf{v}} \cdot \mathbf{t}) \mathbf{t}$

$$
\begin{aligned}
& \int_{\Gamma_{*}}\left(\left(\alpha \frac{\partial \tilde{\mathbf{u}}}{\partial \mathbf{n}} \cdot \mathbf{n}\right)(\mathbf{v} \cdot \mathbf{n})+\left(\alpha \frac{\partial \tilde{\mathbf{u}}}{\partial \mathbf{n}} \cdot \mathbf{t}\right)(\mathbf{v} \cdot \mathbf{t})+\mathbf{n} \cdot\left[q \mathbf{n}-(\mathbf{u} \cdot \mathbf{n}) \mathbf{v}-\alpha \frac{\partial \mathbf{v}}{\partial \mathbf{n}}\right](\tilde{\mathbf{u}} \cdot \mathbf{n})\right) d s \\
& +\int_{\Gamma_{*}} \mathbf{t} \cdot\left[q \mathbf{n}-(\mathbf{u} \cdot \mathbf{n}) \mathbf{v}-\alpha \frac{\partial \mathbf{v}}{\partial \mathbf{n}}\right](\tilde{\mathbf{u}} \cdot \mathbf{t}) d s-\int_{\Gamma_{*}}\left(\alpha \frac{\partial \tilde{\mathbf{u}}}{\partial \mathbf{n}} \cdot \mathbf{t}\right) \mu_{2}-(\tilde{\mathbf{u}} \cdot \mathbf{n}) \mu_{1} d s=0 .
\end{aligned}
$$

(iv) Now choosing variations $\tilde{\mathbf{u}}$ on $\Gamma_{*}$ such that $\tilde{\mathbf{u}} \cdot \mathbf{n}=\tilde{\mathbf{u}} \cdot \mathbf{t}=0, \frac{\partial \tilde{\mathbf{u}}}{\partial \mathbf{n}} \cdot \mathbf{t}=0, \frac{\partial \tilde{\mathbf{u}}}{\partial \mathbf{n}} \cdot \mathbf{n}$ arbitrary, we obtain

$$
\mathbf{v} \cdot \mathbf{n}=0 \text {, on } \Gamma_{*} .
$$

Similarly, the following can be independently shown

$$
\mu_{2}=\mathbf{v} \cdot \mathbf{t}, \quad \mu_{1}=\mathbf{n} \cdot\left[q \mathbf{n}-(\mathbf{u} \cdot \mathbf{n}) \mathbf{v}-\alpha \frac{\partial \mathbf{v}}{\partial \mathbf{n}}\right], \mathbf{t} \cdot\left[q \mathbf{n}-(\mathbf{u} \cdot \mathbf{n}) \mathbf{v}-\alpha \frac{\partial \mathbf{v}}{\partial \mathbf{n}}\right]=0 \text { on } \Gamma_{*} \cdot
$$

(v) Choosing $\tilde{\tilde{\mathbf{u}}} \in C^{\infty}(\Gamma)^{2}$, we can find an extension $\tilde{\mathbf{u}} \in C^{\infty}(\Omega)^{2}$ such that $\left.\tilde{\mathbf{u}}\right|_{\Gamma}=\tilde{\tilde{\mathbf{u}}}$, $\left.\tilde{\mathbf{u}}\right|_{\Gamma \backslash \Gamma_{9}}=0$. Then, we have from (25)

$$
\int_{\Gamma_{9}}\left(\alpha \frac{\partial \tilde{\mathbf{u}}}{\partial \mathbf{n}} \cdot \mathbf{v}-\alpha \frac{\partial \mathbf{v}}{\partial \mathbf{n}} \cdot \tilde{\mathbf{u}}+[q(\tilde{\mathbf{u}} \cdot \mathbf{n})-(\mathbf{u} \cdot \mathbf{n})(\mathbf{v} \cdot \tilde{\mathbf{u}})]-\alpha \frac{\partial \tilde{\mathbf{u}}}{\partial \mathbf{n}} \cdot \boldsymbol{\gamma}\right) d s=0,
$$

from which we obtain

$$
\mathbf{v}=\boldsymbol{\gamma}, \text { and }\left[q \mathbf{n}-(\mathbf{u} \cdot \mathbf{n}) \mathbf{v}-\alpha \frac{\partial \mathbf{v}}{\partial \mathbf{n}}\right]=0, \text { on } \Gamma_{9} .
$$

(vi) Finally setting variations $\tilde{\mathbf{u}}$ such that $\tilde{\mathbf{u}}=0$ on $\Gamma \backslash \Gamma_{f}$, we obtain from (25)

$$
\int_{\Gamma_{f}} \alpha \frac{\partial \tilde{\mathbf{u}}}{\partial \mathbf{n}} \cdot \mathbf{v} d s-\int_{\Gamma_{f}} \tilde{\mathbf{u}}\left(-q \mathbf{n}+\alpha \frac{\partial \mathbf{v}}{\partial \mathbf{n}}\right) d s=0
$$

Using (18) and (24), the first integral in (27) vanishes and we obtain for any arbitrary variation satisfying (18) that

$$
-q \mathbf{n}+\alpha \frac{\partial \mathbf{v}}{\partial \mathbf{n}}=0, \text { on } \Gamma_{f} .
$$

Collecting these assertions, we arrive at the adjoint system (22) where we note that $(\mathbf{u} \cdot \mathbf{n})=0$ on $\Gamma_{f}$.

Note 3.1. It is important to note that (12) is equivalent to the min-max problem [20].

$$
\min _{\min _{\Omega \in \mathscr{U}_{a d}}, \mathbf{u}, p}\left\{\max _{\mathbf{v}, q, \boldsymbol{\eta}, \boldsymbol{\gamma}, \mu_{1}, \mu_{2}} \mathscr{L}\left(\mathbf{u}, p, \mathbf{v}, q, \mu_{1}, \mu_{2}, \boldsymbol{\eta}, \boldsymbol{\gamma}, \Omega\right)\right\} .
$$

Furthermore, from the theory of min-max,

$$
d J_{i}(\mathbf{u}, \Omega) \mathbf{h}=d \mathscr{L}\left(\mathbf{u}, p, \mathbf{v}, q, \mu_{1}, \mu_{2}, \boldsymbol{\eta}, \boldsymbol{\gamma}, \Omega\right) \mathbf{h},
$$

at the solution of the min-max problem (28). 
Next the boundary condition on $\Gamma_{5}$ is homogenized at $\Gamma_{5}=\Gamma_{\mathrm{opt}}$. There exists a unique $\mathbf{g}$ satisfying [8]

$$
\left\{\begin{aligned}
\operatorname{div} \hat{\mathbf{g}}=0, & \text { in } \Omega, \\
\hat{\mathbf{g}}=\mathbf{g}, & \text { on } \Gamma_{\mathrm{opt}}, \\
\hat{\mathbf{g}}=\mathbf{0}, & \text { on } \Gamma \backslash \Gamma_{\mathrm{opt}}
\end{aligned}\right.
$$

Let $\hat{\mathbf{u}}=\mathbf{u}-\hat{\mathbf{g}}$. Then substituting $\hat{\mathbf{u}}$ into (1-3) leads to the following system:

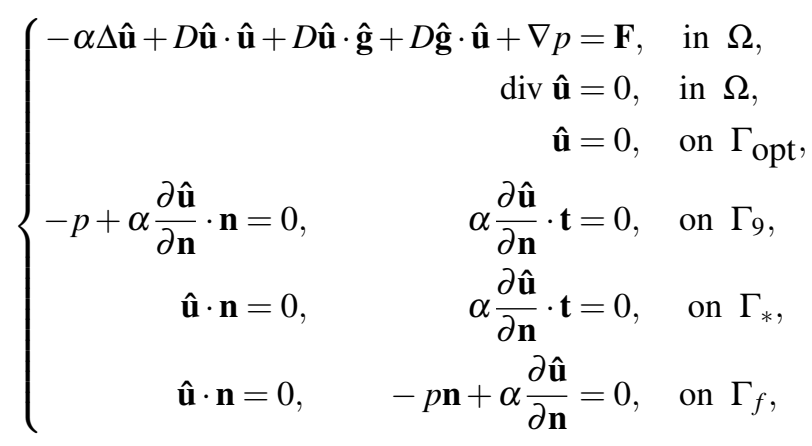

where $\mathbf{F}:=\mathbf{f}+\alpha \Delta \hat{\mathbf{g}}-D \hat{\mathbf{g}} \cdot \hat{\mathbf{g}}$.

For the following result, we require the existence of material derivatives of $\hat{\mathbf{u}}$ and $p$ defined by

$$
\begin{aligned}
& \dot{\hat{\mathbf{u}}}=\lim _{t \rightarrow 0^{+}} \frac{\mathbf{u}_{t} \circ T_{t}-\mathbf{u}}{t}, \\
& \dot{\hat{p}}=\lim _{t \rightarrow 0^{+}} \frac{p_{t} \circ T_{t}-p}{t},
\end{aligned}
$$

provided that the limits exist. For the Navier-Stokes equations, existence of the material derivative is rigorously proved for instance in [18]. Essentially, the existence of the material derivative requires regularity of $\mathbf{f}, \mathbf{g}$ and the domain $\Omega$.

Proposition 3.1. We assume that the material derivatives $\dot{\hat{\mathbf{u}}}$ and $\dot{p}$ exist. Then the shape derivatives $\hat{\mathbf{u}}^{\prime}=\dot{\hat{\mathbf{u}}}-D \hat{\mathbf{u}} \cdot \mathbf{h}$ and $p^{\prime}=\dot{p}-\nabla p \cdot \mathbf{h}$ exist by formal arguments, and they are characterized as the solution of the system

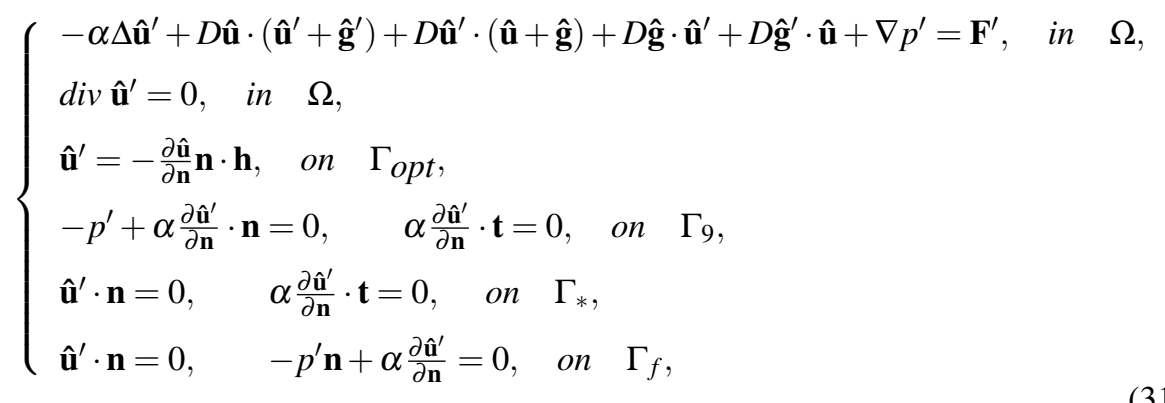

where $\mathbf{F}^{\prime}=\alpha \Delta \hat{\mathbf{g}}^{\prime}-D \hat{\mathbf{g}} \cdot \hat{\mathbf{g}}^{\prime}-D \hat{\mathbf{g}}^{\prime} \cdot \hat{\mathbf{g}}$. Here $\mathbf{h}$ denotes a fixed deformation field which is considered zero on $\Gamma \backslash \Gamma_{\text {opt }}$. 
Before we prove Proposition 3.1, we recall the following essential lemma.

Lemma 3.1. [21] Let $\mathscr{J}=\left[0, \tau_{0}\right]$ with $\tau_{0}$ sufficiently small, $f \in C\left(\mathscr{J}, W^{1,1}(D)\right)$, and assume that $f_{t}(0)$ exists in $L^{1}(D)$. Then

(a) $\left.\frac{d}{d t} \int_{\Omega_{t}} f(t, \mathbf{x}) d \mathbf{x}\right|_{t=0}=\int_{\Omega} f_{t}(0, \mathbf{x}) d \mathbf{x}+\int_{\Gamma} f(0, \mathbf{x}) \mathbf{n} \cdot \mathbf{h} d s$.

(b) Moreover if $f \in C\left(\mathscr{J}, W^{2,1}(D)\right)$, then

$$
\left.\frac{d}{d t} \int_{\Gamma_{t}} f(t, \mathbf{x}) d s_{t}\right|_{t=0}=\int_{\Gamma} f_{t}(0, \mathbf{x}) d s+\int_{\Gamma}\left(\frac{\partial f(0, \mathbf{x})}{\partial \mathbf{n}}+\kappa f(0, \mathbf{x})\right) \mathbf{n} \cdot \mathbf{h} d s,
$$

where $\kappa$ stands for the mean curvature of $\Gamma$.

Proof of Theorem 3.1. Observe that $\hat{\mathbf{u}}$ in (29) satisfies

$\int_{\Omega} \alpha \nabla \hat{\mathbf{u}}: \nabla \boldsymbol{\varphi}+(D \hat{\mathbf{u}} \cdot \hat{\mathbf{u}}+D \hat{\mathbf{u}} \cdot \hat{\mathbf{g}}+D \hat{\mathbf{g}} \cdot \hat{\mathbf{u}}+\nabla p-\mathbf{F}) \boldsymbol{\varphi} d \mathbf{x}=0$, for any $\boldsymbol{\varphi} \in \mathscr{D}\left(\Omega, \mathbb{R}^{2}\right)$.

For $t$ sufficiently small, if $\boldsymbol{\varphi} \in \mathscr{D}\left(\Omega, \mathbb{R}^{2}\right)$, then also $\boldsymbol{\varphi}$ belongs to $\mathscr{D}\left(\Omega_{t}, \mathbb{R}^{2}\right)$ [3], and any solution $\left(\hat{\mathbf{u}}_{t}, p_{t}\right)$ of the Navier-Stokes equations in $\Omega_{t}$ satisfies:

$$
\int_{\Omega_{t}} \alpha \nabla \hat{\mathbf{u}}_{\mathbf{t}}: \nabla \boldsymbol{\varphi}+D \hat{\mathbf{u}}_{\mathbf{t}} \cdot \hat{\mathbf{u}}_{\mathbf{t}} \cdot \boldsymbol{\varphi}+D \hat{\mathbf{u}}_{\mathbf{t}} \cdot \hat{\mathbf{g}}_{t} \cdot \boldsymbol{\varphi}+D \hat{\mathbf{g}}_{t} \cdot \hat{\mathbf{u}}_{\mathbf{t}} \cdot \boldsymbol{\varphi}+\nabla p_{t} \boldsymbol{\varphi}-\mathbf{F} \cdot \boldsymbol{\varphi} d \mathbf{x}_{t}=0 .
$$

Taking the derivative of (32) with respect to $t$ at $t=0$, and using Lemma 3.1(a), we obtain

$$
\begin{aligned}
& \int_{\Omega} \alpha \nabla \hat{\mathbf{u}}^{\prime}: \nabla \boldsymbol{\varphi}+\alpha \nabla \hat{\mathbf{u}}: \nabla \boldsymbol{\varphi}^{\prime}+D \hat{\mathbf{u}}^{\prime} \cdot \hat{\mathbf{u}} \cdot \boldsymbol{\varphi}+D \hat{\mathbf{u}} \cdot \hat{\mathbf{u}}^{\prime} \cdot \boldsymbol{\varphi}+D \hat{\mathbf{u}} \cdot \hat{\mathbf{u}} \cdot \boldsymbol{\varphi}^{\prime}+D \hat{\mathbf{u}}^{\prime} \cdot \hat{\mathbf{g}} \cdot \boldsymbol{\varphi} d \mathbf{x} \\
& +\int_{\Omega} D \hat{\mathbf{u}} \cdot \hat{\mathbf{g}}^{\prime} \cdot \boldsymbol{\varphi}+D \hat{\mathbf{u}} \cdot \hat{\mathbf{g}} \cdot \boldsymbol{\varphi}^{\prime}+D \hat{\mathbf{g}}^{\prime} \cdot \hat{\mathbf{u}} \cdot \boldsymbol{\varphi}+D \hat{\mathbf{g}} \cdot \hat{\mathbf{u}}^{\prime} \cdot \boldsymbol{\varphi}+D \hat{\mathbf{g}} \cdot \hat{\mathbf{u}} \cdot \boldsymbol{\varphi}^{\prime}+\nabla p^{\prime} \boldsymbol{\varphi}+\nabla p \boldsymbol{\varphi}^{\prime} d \mathbf{x} \\
& +\int_{\Gamma_{\mathrm{opt}}}(\alpha \nabla \hat{\mathbf{u}}: \nabla \boldsymbol{\varphi}+D \hat{\mathbf{u}} \cdot \hat{\mathbf{u}} \cdot \boldsymbol{\varphi}+D \hat{\mathbf{u}} \cdot \hat{\mathbf{g}} \cdot \boldsymbol{\varphi}+D \hat{\mathbf{g}} \cdot \hat{\mathbf{u}} \cdot \boldsymbol{\varphi}+\nabla p \boldsymbol{\varphi}-\mathbf{F} \cdot \boldsymbol{\varphi}) \mathbf{n} \cdot \mathbf{h} d s \\
& -\int_{\Omega} \mathbf{F}^{\prime} \cdot \boldsymbol{\varphi}-\mathbf{F} \cdot \boldsymbol{\varphi}^{\prime} d \mathbf{x}=0 .
\end{aligned}
$$

Re-arranging the terms in (33) leads to

$$
\begin{aligned}
& \int_{\Omega} \alpha \nabla \hat{\mathbf{u}}^{\prime}: \nabla \boldsymbol{\varphi}+\left(D \hat{\mathbf{u}} \cdot\left(\hat{\mathbf{u}}^{\prime}+\hat{\mathbf{g}}^{\prime}\right)+D \hat{\mathbf{u}}^{\prime} \cdot(\hat{\mathbf{u}}+\hat{\mathbf{g}})+D \hat{\mathbf{g}} \cdot \hat{\mathbf{u}}^{\prime}+D \hat{\mathbf{g}}^{\prime} \cdot \hat{\mathbf{u}}+\nabla p^{\prime}-\mathbf{F}^{\prime}\right) \boldsymbol{\varphi} d \mathbf{x} \\
& +\int_{\Gamma_{\mathrm{opt}}}(\alpha \nabla \hat{\mathbf{u}}: \nabla \boldsymbol{\varphi}+D \hat{\mathbf{u}} \cdot \hat{\mathbf{u}} \cdot \boldsymbol{\varphi}+D \hat{\mathbf{u}} \cdot \hat{\mathbf{g}} \cdot \boldsymbol{\varphi}+D \hat{\mathbf{g}} \cdot \hat{\mathbf{u}} \cdot \boldsymbol{\varphi}+\nabla p \boldsymbol{\varphi}-\mathbf{F} \cdot \boldsymbol{\varphi}) \mathbf{n} \cdot \mathbf{h} d s \\
& +\int_{\Omega} \alpha \nabla \hat{\mathbf{u}}: \nabla \boldsymbol{\varphi}^{\prime}+(D \hat{\mathbf{u}} \cdot \hat{\mathbf{u}}+D \hat{\mathbf{u}} \cdot \hat{\mathbf{g}}+D \hat{\mathbf{g}} \cdot \hat{\mathbf{u}}+\nabla p-\mathbf{F}) \boldsymbol{\varphi}^{\prime} d \mathbf{x}=0 .
\end{aligned}
$$


Let $\boldsymbol{\varphi}$ be arbitrary and take $\boldsymbol{\varphi}_{t}$, in the form $\boldsymbol{\varphi}_{t},=\boldsymbol{\varphi} \circ T_{t}^{-1} \in \mathscr{D}\left(\Omega_{t}, \mathbb{R}^{2}\right)$. Since $\boldsymbol{\varphi}$, is constant along each streamline $\mathbf{x}+t \mathbf{h}(\mathbf{x}), \mathbf{x} \in \mathbb{R}^{2}$ [9], we have $\dot{\boldsymbol{\varphi}}=0$ in $\Omega$ so that

$$
\boldsymbol{\varphi}^{\prime}=-\nabla \boldsymbol{\varphi} \cdot \mathbf{h}
$$

Applying the Greens formula on the terms $\int_{\Omega} \alpha \nabla \hat{\mathbf{u}}: \nabla \boldsymbol{\varphi}^{\prime} d \mathbf{x}$ and $\int_{\Omega} \alpha \nabla \hat{\mathbf{u}}^{\prime}: \nabla \boldsymbol{\varphi} d \mathbf{x}$, we obtain

$$
-\int_{\Omega} \alpha \nabla \hat{\mathbf{u}}: \nabla(\nabla \boldsymbol{\varphi} \cdot \mathbf{h}) d \mathbf{x}=-\int_{\Gamma_{\mathrm{opt}}} \alpha \frac{\partial \hat{\mathbf{u}}}{\partial \mathbf{n}} \nabla \boldsymbol{\varphi} \cdot \mathbf{h} d s-\int_{\Omega} \alpha \Delta \hat{\mathbf{u}} \boldsymbol{\varphi}^{\prime} d \mathbf{x}
$$

and

$$
\int_{\Omega} \alpha \nabla \hat{\mathbf{u}}^{\prime}: \nabla \boldsymbol{\varphi} d \mathbf{x}=\int_{\Gamma_{\mathrm{opt}}} \alpha \frac{\partial \hat{\mathbf{u}}^{\prime}}{\partial \mathbf{n}} \boldsymbol{\varphi} d s-\int_{\Omega} \alpha \Delta \hat{\mathbf{u}}^{\prime} \boldsymbol{\varphi} d \mathbf{x}
$$

respectively, making use of (35) again. Note that

$$
\begin{aligned}
& \boldsymbol{\varphi}^{\prime}=-\frac{\partial \boldsymbol{\varphi}}{\partial \mathbf{n}} \mathbf{h} \cdot \mathbf{n}, \quad-\alpha \frac{\partial \hat{\mathbf{u}}}{\partial \mathbf{n}} \nabla \boldsymbol{\varphi} \cdot \mathbf{h}=-\alpha \frac{\partial \hat{\mathbf{u}}}{\partial \mathbf{n}} \frac{\partial \boldsymbol{\varphi}}{\partial \mathbf{n}} \mathbf{h} \cdot \mathbf{n} \text { and } \\
& \alpha \nabla \hat{\mathbf{u}}: \nabla \boldsymbol{\varphi}(\mathbf{h} \cdot \mathbf{n})=\alpha \frac{\partial \hat{\mathbf{u}}}{\partial \mathbf{n}} \frac{\partial \boldsymbol{\varphi}}{\partial \mathbf{n}} \mathbf{h} \cdot \mathbf{n}
\end{aligned}
$$

since $\boldsymbol{\varphi} \in \mathscr{D}\left(\Omega, \mathbb{R}^{2}\right)$, yielding $\partial \boldsymbol{\varphi} / \partial \mathbf{s}=0$ on $\Gamma_{\text {opt }}$, where $\partial / \partial \mathbf{s}=0$ stands for the derivative along $\Gamma_{\text {opt }}$. From (29.1), (34), (36), (37), (38) and the fact that $\boldsymbol{\varphi}$ has compact support, the second and third expressions in (34) vanish such that we obtain

$$
\int_{\Omega}\left(-\alpha \Delta \hat{\mathbf{u}}^{\prime}+D \hat{\mathbf{u}} \cdot\left(\hat{\mathbf{u}}^{\prime}+\hat{\mathbf{g}}^{\prime}\right)+D \hat{\mathbf{u}}^{\prime} \cdot(\hat{\mathbf{u}}+\hat{\mathbf{g}})+D \hat{\mathbf{g}} \cdot \hat{\mathbf{u}}^{\prime}+D \hat{\mathbf{g}}^{\prime} \cdot \hat{\mathbf{u}}+\nabla p^{\prime}-\mathbf{F}^{\prime}\right) \cdot \boldsymbol{\varphi} d \mathbf{x}=0 .
$$

By density arguments, we obtain

$$
-\alpha \Delta \hat{\mathbf{u}}^{\prime}+D \hat{\mathbf{u}} \cdot\left(\hat{\mathbf{u}}^{\prime}+\hat{\mathbf{g}}^{\prime}\right)+D \hat{\mathbf{u}}^{\prime} \cdot(\hat{\mathbf{u}}+\hat{\mathbf{g}})+D \hat{\mathbf{g}} \cdot \hat{\mathbf{u}}^{\prime}+D \hat{\mathbf{g}}^{\prime} \cdot \hat{\mathbf{u}}+\nabla p^{\prime}=\mathbf{F}^{\prime},
$$

which gives the first equation in (31). Since $\hat{\mathbf{u}}=0$ on $\Gamma_{\text {opt }}$, we have

$$
\int_{\Gamma_{\mathrm{opt}}} \hat{\mathbf{u}} \cdot \boldsymbol{\varphi} d s=0, \text { for all } \boldsymbol{\varphi} \in C^{1}\left(\bar{D} ; \mathbb{R}^{2}\right)
$$

On $\Gamma_{\mathrm{opt}, t},(39)$ becomes

$$
\int_{\Gamma_{\mathrm{opt}, t}} \hat{\mathbf{u}}_{\mathbf{t}} \cdot \boldsymbol{\varphi} d s_{t}=0, \text { for all } \boldsymbol{\varphi} \in C^{1}\left(\bar{D} ; \mathbb{R}^{2}\right)
$$

Taking the derivative of equation (40) with respect to $t$ at $t=0$, and using Lemma 3.1(b), we obtain

$$
\int_{\Gamma_{\mathrm{opt}}} \hat{\mathbf{u}}^{\prime} \cdot \boldsymbol{\varphi}+\boldsymbol{\varphi}^{\prime} \cdot \hat{\mathbf{u}} d s+\int_{\Gamma_{\mathrm{opt}}}\left[\frac{\partial}{\partial \mathbf{n}}(\hat{\mathbf{u}} \cdot \boldsymbol{\varphi})+\kappa \hat{\mathbf{u}} \cdot \boldsymbol{\varphi}\right] \mathbf{n} \cdot \mathbf{h} d s=0,
$$


where $\kappa$ is the curvature of the boundary. Using the expression for $\boldsymbol{\varphi}^{\prime}$ in (38), equation (41) simplifies to

$$
\int_{\Gamma_{\mathrm{opt}}}\left(\hat{\mathbf{u}}^{\prime} \cdot \boldsymbol{\varphi}+\frac{\partial \hat{\mathbf{u}}}{\partial \mathbf{n}} \cdot \boldsymbol{\varphi n} \cdot \mathbf{h}\right) d s=0 .
$$

Again by density arguments, we have that

$$
\hat{\mathbf{u}}^{\prime}=-\frac{\partial \hat{\mathbf{u}}}{\partial \mathbf{n}} \mathbf{n} \cdot \mathbf{h}, \text { on } \Gamma_{\mathrm{opt}},
$$

which gives the third equation in (31). Since $\mathbf{h}$ is assumed to be zero on the other boundaries, derivation of the other boundary conditions easily follow in a similar way.

Since $\mathbf{g}$ is independent of the shape, the shape derivative $\mathbf{u}^{\prime}$ of the solution $\mathbf{u}$ of the original Navier-Stokes system (1-3) is given by $\hat{\mathbf{u}}^{\prime}=\mathbf{u}^{\prime}-\hat{\mathbf{g}}^{\prime}$, where $\hat{\mathbf{g}}^{\prime}=\mathbf{g}^{\prime}=0$ on $\Gamma_{\text {opt }}$. Therefore, we obtain the following corollary by substituting $\hat{\mathbf{u}}^{\prime}=\mathbf{u}^{\prime}-\hat{\mathbf{g}}^{\prime}$ and $\hat{\mathbf{u}}=\mathbf{u}-\hat{\mathbf{g}}$ into (31).

Corollary 3.1. The shape derivative $\mathbf{u}^{\prime}$ of system (1-3) satisfies the following system

$$
\left\{\begin{aligned}
-\alpha \Delta \mathbf{u}^{\prime}+D \mathbf{u} \cdot \mathbf{u}^{\prime}+D \mathbf{u}^{\prime} \cdot \mathbf{u}+\nabla p^{\prime} & =0, \\
\operatorname{div} \mathbf{u}^{\prime} & =0, \text { in } \Omega, \\
\mathbf{u}^{\prime} & =-\frac{\partial(\mathbf{u}-\mathbf{g})}{\partial \mathbf{n}} \mathbf{n} \cdot \mathbf{h}, \text { on } \Gamma_{\text {opt }}, \\
-p^{\prime}+\alpha \frac{\partial \mathbf{u}^{\prime}}{\partial \mathbf{n}} \cdot \mathbf{n}=0, \quad \alpha \frac{\partial \mathbf{u}^{\prime}}{\partial \mathbf{n}} \cdot \mathbf{t} & =0, \text { on } \Gamma_{9}, \\
\mathbf{u}^{\prime} \cdot \mathbf{n}=0, \quad \alpha \frac{\partial \mathbf{u}^{\prime}}{\partial \mathbf{n}} \cdot \mathbf{t} & =0, \text { on } \Gamma_{*}, \\
\mathbf{u}^{\prime} \cdot \mathbf{n}=0, \quad\left(-p^{\prime} \mathbf{n}+\alpha \frac{\partial \mathbf{u}^{\prime}}{\partial \mathbf{n}}\right) & =0, \text { on } \Gamma_{f} .
\end{aligned}\right.
$$

Remark 3.1. We assume that the free boundary is disjoint from the control boundary and fixed during the optimization step. This implies that not only that $\mathbf{u}^{\prime} \cdot \mathbf{n}=0$, on $\Gamma_{f}$, but also $\mathbf{u}^{\prime} \cdot \mathbf{t}=0$, on $\Gamma_{f}$. Consequently, the following conditions

$$
\begin{aligned}
\left(-p^{\prime} \mathbf{n}+\alpha \mathbf{n} \cdot \nabla \mathbf{u}^{\prime}\right)=0, & \text { on } \Gamma_{f}, \\
\mathbf{u}^{\prime} \cdot \mathbf{n}=0, \quad \mathbf{u}^{\prime} \cdot \mathbf{t}=0, & \text { on } \Gamma_{f},
\end{aligned}
$$

hold.

In the sequel, the following lemma will be utilized.

Lemma 3.2. The shape derivative $\mathbf{u}^{\prime}$ in (42) satisfies

$$
\mathbf{u}^{\prime} \cdot \mathbf{n}=0, \text { on } \Gamma_{\text {opt }}
$$


Proof. Using $\mathbf{u}^{\prime}=-\frac{\partial(\mathbf{u}-\mathbf{g})}{\partial \mathbf{n}} \mathbf{n} \cdot \mathbf{h}$ on $\Gamma_{\text {opt }}$, using the tangential divergence formula [21], we have on $\Gamma_{\text {opt }}$ that

$$
\mathbf{u}^{\prime} \cdot \mathbf{n}=-\frac{\partial(\mathbf{u}-\mathbf{g})}{\partial \mathbf{n}} \cdot \mathbf{n}(\mathbf{h}, \mathbf{n})=\left.\operatorname{div}(\mathbf{u}-\hat{\mathbf{g}})(\mathbf{h}, \mathbf{n})\right|_{\Gamma_{\text {opt }}}-\operatorname{div}_{\Gamma_{\text {opt }}}(\mathbf{u}-\mathbf{g})(\mathbf{h}, \mathbf{n}) .
$$

Using $\mathbf{u}-\mathbf{g}=0$ on $\Gamma_{\text {opt }}$, as well as considering the fact that we are using divergence free fields, the expression in (43) vanishes.

\subsection{Gradients of cost functionals}

The goal here is to find the Eulerian derivatives of $J_{i}(\mathbf{u}, \Omega), i=1, \ldots, 3$, in the direction of the deformation vector field $\mathbf{h}$.

Theorem 3.2. Let $\mathbf{u}_{d}$ the desired flow field, and $\mathbf{h}$ a fixed vector field. Then the shape gradient $\nabla j_{1} \mathbf{n}$ of the cost functional $J_{1}$ can be expressed as

$$
\nabla j_{1} \mathbf{n}=\left[\frac{1}{2}\left|\mathbf{u}-\mathbf{u}_{d}\right|^{2}+\alpha \frac{\partial(\mathbf{u}-\mathbf{g})}{\partial \mathbf{n}} \cdot \frac{\partial \mathbf{v}}{\partial \mathbf{n}}\right] \mathbf{n},
$$

where all expressions are evaluated on $\Gamma_{o p t}$, and the adjoint state $\mathbf{v}$ satisfies (22) with $j_{1}^{\prime}(\mathbf{u})=\left(\mathbf{u}-\mathbf{u}_{d}\right)$.

Proof. Since $J_{1}(\mathbf{u}, \Omega)$ is differentiable with respect to $\mathbf{u}$, by Lemma 3.1 we obtain the Eulerian derivative of $J_{1}(\mathbf{u}, \Omega)$ with respect to $\Omega$,

$$
d J_{1}(\mathbf{u}, \Omega) \mathbf{h}=\int_{\Omega}\left(\mathbf{u}-\mathbf{u}_{d}\right) \mathbf{u}^{\prime} d \mathbf{x}+\int_{\Gamma_{\mathrm{opt}}} \frac{1}{2}\left|\mathbf{u}-\mathbf{u}_{d}\right|^{2} \mathbf{n} \cdot \mathbf{h} d s
$$

Testing system (42) with the adjoint variable $(\mathbf{v}, q)$ and utilizing the adjoint system (22), we have

$$
0=\int_{\Omega}\left(\left(-\alpha \Delta \mathbf{u}^{\prime}+D \mathbf{u} \cdot \mathbf{u}^{\prime}+D \mathbf{u}^{\prime} \cdot \mathbf{u}+\nabla p^{\prime}\right) \cdot \mathbf{v}-\left(\operatorname{div} \mathbf{u}^{\prime}\right) \cdot q\right) d \mathbf{x}
$$

Applying Greens formula to equation (46) gives

$$
\begin{aligned}
0= & \int_{\Omega}\left[\left(-\alpha \Delta \mathbf{v}-D \mathbf{v} \cdot \mathbf{u}+[D \mathbf{u}]^{t} \cdot \mathbf{v}+\nabla q\right) \cdot \mathbf{u}^{\prime}-(\operatorname{div} \mathbf{v}) \cdot p^{\prime}\right] d \mathbf{x} \\
& -\int_{\Gamma} \mathbf{u}^{\prime}\left(q \mathbf{n}-(\mathbf{u} \cdot \mathbf{n}) \mathbf{v}-\alpha \frac{\partial \mathbf{v}}{\partial \mathbf{n}}\right) d s-\int_{\Gamma}\left(-p^{\prime} \mathbf{n}+\alpha \frac{\partial \mathbf{u}^{\prime}}{\partial \mathbf{n}}\right) \mathbf{v} d s
\end{aligned}
$$

Since $(\mathbf{v}, q)$ satisfies the adjoint system (22), we have

$$
0=\int_{\Omega}\left(\mathbf{u}-\mathbf{u}_{d}\right) \cdot \mathbf{u}^{\prime} d \mathbf{x}-\int_{\Gamma} \mathbf{u}^{\prime}\left(q \mathbf{n}-(\mathbf{u} \cdot \mathbf{n}) \mathbf{v}-\alpha \frac{\partial \mathbf{v}}{\partial \mathbf{n}}\right) d s-\int_{\Gamma}\left(-p^{\prime} \mathbf{n}+\alpha \frac{\partial \mathbf{u}^{\prime}}{\partial \mathbf{n}}\right) \mathbf{v} d s
$$

If we write the boundary terms in (48) in component form:

$$
\begin{aligned}
& \int_{\Gamma}\left[\left(\mathbf{u}^{\prime} \cdot \mathbf{n}\right)\left(q \mathbf{n}-(\mathbf{u} \cdot \mathbf{n}) \mathbf{v}-\alpha \frac{\partial \mathbf{v}}{\partial \mathbf{n}}\right) \cdot \mathbf{n}+\left(\mathbf{u}^{\prime} \cdot \mathbf{t}\right)\left(q \mathbf{n}-(\mathbf{u} \cdot \mathbf{n}) \mathbf{v}-\alpha \frac{\partial \mathbf{v}}{\partial \mathbf{n}}\right) \cdot \mathbf{t}\right] d s \\
& \int_{\Gamma}\left[(\mathbf{v} \cdot \mathbf{n})\left(-p^{\prime} \mathbf{n}+\alpha \frac{\partial \mathbf{u}^{\prime}}{\partial \mathbf{n}}\right) \cdot \mathbf{n}+(\mathbf{v} \cdot \mathbf{t})\left(-p^{\prime} \mathbf{n}+\alpha \frac{\partial \mathbf{u}^{\prime}}{\partial \mathbf{n}}\right) \cdot \mathbf{t}\right] d s
\end{aligned}
$$


then it is easy to see that these terms in (49) vanish on $\Gamma_{9}, \Gamma_{*}$ and $\Gamma_{f}$, due to (22) and (42). Therefore, equation (48) becomes

$$
\int_{\Omega}\left(\mathbf{u}-\mathbf{u}_{d}\right) \cdot \mathbf{u}^{\prime} d \mathbf{x}=-\int_{\Gamma_{\mathrm{opt}}}\left(\alpha \frac{\partial \mathbf{v}}{\partial \mathbf{n}}-q \mathbf{n}\right) \mathbf{u}^{\prime} d s .
$$

The term $(q \mathbf{n}) \mathbf{u}^{\prime}$ in (50) vanishes on $\Gamma_{\text {opt }}$, due to Lemma 3.2. Hence, using (42), we obtain the Eulerian derivative from (45):

$$
d J_{1}(\mathbf{u}, \Omega) \mathbf{h}=\int_{\Gamma_{\mathrm{opt}}}\left(\frac{1}{2}\left|\mathbf{u}-\mathbf{u}_{d}\right|^{2}+\alpha \frac{\partial(\mathbf{u}-\mathbf{g})}{\partial \mathbf{n}} \cdot \frac{\partial \mathbf{v}}{\partial \mathbf{n}}\right) \mathbf{n} \cdot \mathbf{h} d s
$$

Since the mapping $\mathbf{h} \mapsto d J_{1}(\mathbf{u}, \Omega) \mathbf{h}$ is linear and continuous, we get the expression for the shape gradient (44).

Theorem 3.3. Let $\mathbf{h}$ be a fixed vector field. Then the shape gradient $\nabla j_{2} \mathbf{n}$ of the cost functional $J_{2}(\mathbf{u}, \Omega)$ can be expressed as

$$
\nabla j_{2} \mathbf{n}=\left[\frac{1}{2}|\operatorname{curl} \mathbf{u}|^{2}+\frac{\partial(\mathbf{u}-\mathbf{g})}{\partial \mathbf{n}} \cdot\left(\alpha \frac{\partial \mathbf{v}}{\partial \mathbf{n}}-\tau \text { curl } \mathbf{u}\right)\right] \mathbf{n}
$$

where all expressions are evaluated on $\Gamma_{o p t}$, and the adjoint state $\mathbf{v}$ satisfies (22) with $j_{2}^{\prime}(\mathbf{u})=-\Delta \mathbf{u}$.

Proof. Since $J_{2}(\mathbf{u}, \Omega)$ is differentiable with respect to $\mathbf{u}$, by Lemma 3.1, we obtain the Eulerian derivative of $J_{2}(\mathbf{u}, \Omega)$ with respect to $t$,

$$
d J_{2}(\mathbf{u}, \Omega) \mathbf{h}=\int_{\Omega} \operatorname{curl} \mathbf{u} \operatorname{curl} \mathbf{u}^{\prime} d \mathbf{x}+\int_{\Gamma_{\mathrm{opt}}} \frac{1}{2}|\operatorname{curl} \mathbf{u}|^{2} \mathbf{n} \cdot \mathbf{h} d s .
$$

Utilizing arguments in (46), (47), we have for the solution $(\mathbf{v}, q)$ of the adjoint system (22) with $j_{2}^{\prime}(\mathbf{u})=-\Delta \mathbf{u}$, that

$$
0=\int_{\Omega}-\Delta \mathbf{u} \cdot \mathbf{u}^{\prime} d \mathbf{x}-\int_{\Gamma} \mathbf{u}^{\prime}\left(q \mathbf{n}-(\mathbf{u} \cdot \mathbf{n}) \mathbf{v}-\alpha \frac{\partial \mathbf{v}}{\partial \mathbf{n}}\right) d s-\int_{\Gamma}\left(-p^{\prime} \mathbf{n}+\alpha \frac{\partial \mathbf{u}^{\prime}}{\partial \mathbf{n}}\right) \mathbf{v} d s
$$

Using similar arguments as before, we have

$$
\int_{\Omega} \Delta \mathbf{u} \cdot \mathbf{u}^{\prime} d \mathbf{x}=\int_{\Gamma_{\mathrm{opt}}}\left(\alpha \frac{\partial \mathbf{v}}{\partial \mathbf{n}}-q \mathbf{n}\right) \mathbf{u}^{\prime} d s .
$$

The term $(q \mathbf{n}) \mathbf{u}^{\prime}$ vanishes on $\Gamma_{\text {opt }}$, due to Lemma 3.2. Thus, (54) becomes

$$
\int_{\Omega} \Delta \mathbf{u} \cdot \mathbf{u}^{\prime} d \mathbf{x}=-\int_{\Gamma_{\mathrm{opt}}} \alpha \frac{\partial(\mathbf{u}-\mathbf{g})}{\partial \mathbf{n}} \cdot \frac{\partial \mathbf{v}}{\partial \mathbf{n}} \mathbf{n} \cdot \mathbf{h} d s .
$$

Using Greens formula [17], we have that

$$
\int_{\Omega} \operatorname{curl} \mathbf{u} \operatorname{curl} \mathbf{u}^{\prime} d \mathbf{x}=-\int_{\Omega} \Delta \mathbf{u} \cdot \mathbf{u}^{\prime} d \mathbf{x}+\int_{\Gamma}\left(\mathbf{u}^{\prime} \cdot \boldsymbol{\tau}\right) \operatorname{curl} \mathbf{u} d s .
$$


Utilizing (55) and (42), we obtain

$$
\int_{\Omega} \operatorname{curl} \mathbf{u} \operatorname{curl} \mathbf{u}^{\prime} d \mathbf{x}=\int_{\Gamma_{\mathrm{opt}}} \frac{\partial(\mathbf{u}-\mathbf{g})}{\partial \mathbf{n}} \cdot\left(\alpha \frac{\partial \mathbf{v}}{\partial \mathbf{n}}-\tau(\operatorname{curl} \mathbf{u})\right) \mathbf{n} \cdot \mathbf{h} d s .
$$

Hence, we obtain the Eulerian derivative from (53):

$$
d J_{2}(\mathbf{u}, \Omega) \mathbf{h}=\int_{\Gamma_{\mathrm{opt}}}\left(\frac{1}{2}|\operatorname{curl} \mathbf{u}|^{2}+\frac{\partial(\mathbf{u}-\mathbf{g})}{\partial \mathbf{n}} \cdot\left(\alpha \frac{\partial \mathbf{v}}{\partial \mathbf{n}}-\tau(\operatorname{curl} \mathbf{u})\right)\right) \mathbf{n} \cdot \mathbf{h} d s
$$

Since the mapping $\mathbf{h} \mapsto d J_{2}(\mathbf{u}, \Omega) \mathbf{h}$ is linear and continuous, we get the expression for the shape gradient (52).

Theorem 3.4. Let $\mathbf{h}$ be a fixed vector field. Then the shape gradient $\nabla j_{3} \mathbf{n}$ of the cost functional $J_{3}(\mathbf{u}, \Omega)$ can be expressed as

$$
\nabla j_{3} \mathbf{n}=\left[g_{3}(\operatorname{det} \nabla \mathbf{u})+\frac{\partial(\mathbf{u}-\mathbf{g})}{\partial \mathbf{n}} \cdot\left(\alpha \frac{\partial \mathbf{v}}{\partial \mathbf{n}}-P(\mathbf{u})\right)\right] \mathbf{n},
$$

where all expressions are evaluated on $\Gamma_{\text {opt }}$, and the adjoint state $\mathbf{v}$ satisfies (22) with $j_{3}^{\prime}(\mathbf{u})=R(\mathbf{u})$, where

$$
R(\mathbf{u})=\left(\begin{array}{c}
-\operatorname{curl}\left(\vartheta \nabla u_{2}\right) \\
\operatorname{curl}\left(\vartheta \nabla u_{1}\right)
\end{array}\right), \quad P(\mathbf{u})=\left(\begin{array}{c}
\vartheta\left(\frac{\partial u_{2}}{\partial x_{2}} n_{x_{1}}-\frac{\partial u_{2}}{\partial x_{1}} n_{x_{2}}\right) \\
\vartheta\left(\frac{\partial u_{1}}{\partial x_{1}} n_{x_{2}}-\frac{\partial u_{1}}{\partial x_{2}} n_{x_{1}}\right)
\end{array}\right)
$$

$\vartheta=g_{3}^{\prime}(\operatorname{det} \nabla \mathbf{u})$ and $\mathbf{u}=\left(u_{1}, u_{2}\right)^{T}$.

Proof. Since $J_{3}(\mathbf{u}, \Omega)$ is differentiable with respect to $\mathbf{u}$, by Lemma 3.1, we obtain the Eulerian derivative of $J_{3}(\mathbf{u}, \Omega)$ with respect to $t$,

$$
d J_{3}(\mathbf{u}, \Omega) \mathbf{h}=\int_{\Omega} \mathscr{B} d \mathbf{x}+\int_{\Gamma_{\text {opt }}} g_{3}(\operatorname{det} \nabla \mathbf{u}) \mathbf{n} \cdot \mathbf{h} d s
$$

where $\mathscr{B}=g_{3}^{\prime}(\operatorname{det} \nabla \mathbf{u})\left(\frac{\partial u_{1}}{\partial x_{1}} \frac{\partial u_{2}^{\prime}}{\partial x_{2}}+\frac{\partial u_{1}^{\prime}}{\partial x_{1}} \frac{\partial u_{2}}{\partial x_{2}}-\frac{\partial u_{2}}{\partial x_{1}} \frac{\partial u_{1}^{\prime}}{\partial x_{2}}-\frac{\partial u_{2}^{\prime}}{\partial x_{1}} \frac{\partial u_{1}}{\partial x_{2}}\right), u_{1}^{\prime}(\mathbf{x}):=\left.\frac{\partial u_{1, t}}{\partial t}(\mathbf{x})\right|_{t=0}$, $u_{2}^{\prime}(\mathbf{x}):=\left.\frac{\partial u_{2, t}}{\partial t}(\mathbf{x})\right|_{t=0}$ and $\mathbf{u}^{\prime}(\mathbf{x})=\left(u_{1}^{\prime}(\mathbf{x}), u_{2}^{\prime}(\mathbf{x})\right)^{T}$.

Using integration by parts, the first term in (58) can be written as

$$
\int_{\Omega} \mathscr{B} d \mathbf{x}=\int_{\Omega} R(\mathbf{u}) \cdot \mathbf{u}^{\prime} d \mathbf{x}+\int_{\Gamma_{\mathrm{opt}}} P(\mathbf{u}) \cdot \mathbf{u}^{\prime} d s .
$$

Following the arguments in (46), (47), we have for the solution $(\mathbf{v}, q)$ of the adjoint system (22) with $j_{3}^{\prime}(\mathbf{u})=R(\mathbf{u})$, that

$$
0=\int_{\Omega} R(\mathbf{u}) \cdot \mathbf{u}^{\prime} d \mathbf{x}-\int_{\Gamma_{\mathrm{opt}}}\left(\mathbf{u}^{\prime}\left(q \mathbf{n}-(\mathbf{u} \cdot \mathbf{n}) \mathbf{v}-\alpha \frac{\partial \mathbf{v}}{\partial \mathbf{n}}\right)-\left(-p^{\prime} \mathbf{n}+\alpha \frac{\partial \mathbf{u}^{\prime}}{\partial \mathbf{n}}\right) \mathbf{v}\right) d s
$$


Using similar arguments as in (49), we obtain

$$
\int_{\Omega} R(\mathbf{u}) \cdot \mathbf{u}^{\prime} d \mathbf{x}=\int_{\Gamma_{\mathrm{opt}}}\left(\alpha \frac{\partial \mathbf{v}}{\partial \mathbf{n}}-q \mathbf{n}\right) \mathbf{u}^{\prime} d s .
$$

Utilizing (42), we obtain the Eulerian derivative from (58):

$$
d J_{3}(\mathbf{u}, \Omega) \mathbf{h}=\int_{\Gamma_{\mathrm{opt}}}\left(g_{3}(\operatorname{det} \nabla \mathbf{u})+\frac{\partial \mathbf{u}}{\partial \mathbf{n}} \cdot\left(\alpha \frac{\partial \mathbf{v}}{\partial \mathbf{n}}-P(\mathbf{u})\right)\right) \mathbf{n} \cdot \mathbf{h} d s
$$

Since the mapping $\mathbf{h} \mapsto d J_{3}(\mathbf{u}, \Omega) \mathbf{h}$ is linear and continuous, we get the expression for the shape gradient (57).

Remark 3.2. Since Remark 3.1 holds, the integrals in (49), evaluated on $\Gamma_{f}$, vanish irrespective of which boundary condition is posed on $\Gamma_{f}$ for the adjoint problem. Therefore, it suffices to consider only one of the conditions:

$$
\begin{aligned}
{[q \cdot \mathbf{n}-(\mathbf{u} \cdot \mathbf{n}) \mathbf{v}-\alpha \nabla \mathbf{v} \cdot \mathbf{n}] } & =0, \text { on } \Gamma_{f}, \\
\mathbf{v} \cdot \mathbf{n} & =0, \text { on } \Gamma_{f},
\end{aligned}
$$

for the adjoint problem, so that the expressions for the shape derivatives of $J_{i}, i=1,2,3$ in (51), (56), and (59) are satisfied. In this work we chose to solve the following adjoint system

$$
\left\{\begin{array}{l}
-\alpha \Delta \mathbf{v}-(\mathbf{u} \cdot \nabla) \mathbf{v}+[\nabla \mathbf{u}] \cdot \mathbf{v}+\nabla q=J_{i}^{\prime}(\mathbf{u}), \text { in } \Omega \\
\operatorname{div} \mathbf{v}=0, \text { in } \Omega, \\
\mathbf{v} \cdot \mathbf{n}=0, \mathbf{t} \cdot[q \cdot \mathbf{n}-(\mathbf{u} \cdot \mathbf{n}) \mathbf{v}-\alpha \nabla \mathbf{v} \cdot \mathbf{n}]=0, \text { on } \Gamma_{*}, \\
\mathbf{v}=0, \text { on } \Gamma_{5}, \\
q \cdot \mathbf{n}-(\mathbf{u} \cdot \mathbf{n}) \mathbf{v}-\alpha \nabla \mathbf{v} \cdot \mathbf{n}=0, \text { on } \Gamma_{f} \\
q \cdot \mathbf{n}-(\mathbf{u} \cdot \mathbf{n}) \mathbf{v}-\alpha \nabla \mathbf{v} \cdot \mathbf{n}=0, \text { on } \Gamma_{9}
\end{array}\right.
$$

\section{Algorithmic realization and numerical examples}

In this section we consider two optimization problems. Firstly, the optimization of the shape on the inflow portion $\Gamma_{5}$ of the flow domain $\Omega$ in Figure 1. Figure 3 (a) shows the geometrical set up of this portion, not drawn to scale. Secondly, the optimization of the shape of the boundaries $\Gamma_{4}$ and $\Gamma_{6}$ of the domain $\Omega$ (Figure $3(\mathrm{~b})$ ). In both cases, we let $\Gamma_{\text {opt }}$ be represented by the graph of functions $\alpha$ chosen from appropriate sets of admissible graphs. In particular, for the first problem, we let $\alpha \in \mathscr{O}$, where

$$
\mathscr{O}=\left\{\alpha \in C^{1,1}([a, b]) \mid 0<\alpha_{\text {min }} \leq \alpha\left(x_{1}\right) \leq \alpha_{\max }, x_{1} \in[a, b]\right\} .
$$

In the second problem, we let $\alpha \in \mathscr{O}_{i}, i=1,2$, where

$$
\begin{aligned}
& \mathscr{O}_{1}=\left\{\alpha_{1} \in C^{1,1}([c, d]) \mid \alpha_{\{\min , 1\}} \leq \alpha_{1}\left(x_{2}\right) \leq \alpha_{\{\max , 1\}}, x_{2} \in[c, d]\right\} \\
& \mathscr{O}_{2}=\left\{\alpha_{2} \in C^{1,1}([c, d]) \mid \alpha_{\{\min , 2\}} \leq \alpha_{2}\left(x_{2}\right) \leq \alpha_{\{\max , 2\}}, x_{2} \in[c, d]\right\} .
\end{aligned}
$$




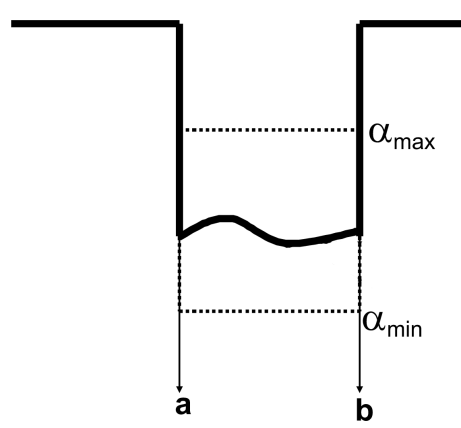

(a) Problem 1

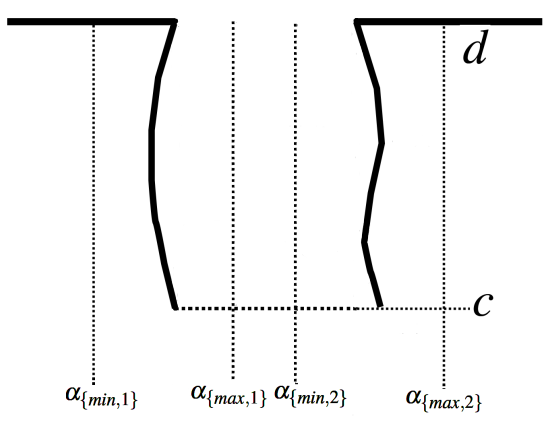

(b) Problem 2

Figure 3: Admissible domains

Consequently, the sets of admissible domains for problems 1 and 2 are defined as

$$
\mathscr{U}_{a d}=\{\Omega(\alpha) \mid \alpha \in \mathscr{O}\}
$$

and

$$
\mathscr{U}_{a d}=\left\{\Omega(\alpha) \mid \alpha_{1} \in \mathscr{O}_{1}, \alpha_{2} \in \mathscr{O}_{2}\right\}
$$

respectively. We solve the optimization problems in their original setting, i.e., we find a solution to the state problem (1-3) first and then proceed to the upper level represented by the minimization of the cost functionals (9-11). On the minimization level, we utilize the boundary variation technique [2]. We remark here that the form of the deformation field $\mathbf{h}$ realizing shape variations depends on how shapes of admissible domains are parametrized [9]. Therefore, for the family of domains shown in Figures $3(\mathrm{a})$ and $3(\mathrm{~b})$, it is natural to take the deformation fields of the form $\mathbf{h}=\left(0, h_{2}\right)$ and $\mathbf{h}=\left(h_{1}, 0\right)$, respectively. Here, $h_{i}$ corresponds to the variation $\delta \alpha=\tilde{\alpha}-\alpha$, where $\tilde{\alpha} \in \mathscr{O}$ is a function that determines $\Gamma_{\text {opt }}(\tilde{\alpha})$ after deformation of $\Gamma_{\text {opt }}(\alpha)$ by $\delta \alpha$. Moreover, $\delta \alpha$ is defined on the boundary $\Gamma_{\text {opt }}$ and can be taken as one of the negative gradients according to (44), (52) and (57). In fact, for the geometries depicted in Figure 3, only one of the coordinates of $\nabla j_{i} \mathbf{n}$ is nontrivial. However, to avoid ambiguity with (14)-(15), we must extend this variation such that it is defined over the entire domain. This is done by Algorithm 2 which extends the deformation field $\mathbf{h}$ over the entire domain. It provides a descent direction for the cost functional $J_{i}, i=1,2,3$ :

$$
d J_{i}(\mathbf{u}, \Omega) \mathbf{h}=\int_{\Gamma_{\mathrm{opt}}} \nabla J_{i} \mathbf{n} \cdot \mathbf{h} d s=-\int_{\Omega}|\nabla \mathbf{h}|^{2}+|\mathbf{h}|^{2} d \mathbf{x}<0 .
$$

Remark 4.1. In the above form, Algorithm 2 does not provide for the inequality constraints in $\mathscr{U}_{a d}$. To realize these constraints, a penalty approach is used [13]. Furthermore, no gradient constraints on the curve are imposed and therefore possible boundary oscillations of $\Gamma_{\text {opt }}$ can occur. The extension of $\mathbf{h}$ on the basis of (66) is also regularizing. If the Neumann boundary condition in (66) is replaced by a Dirichlet condition, then the regularization is insufficient and undesired oscillations of the shapes occur. 


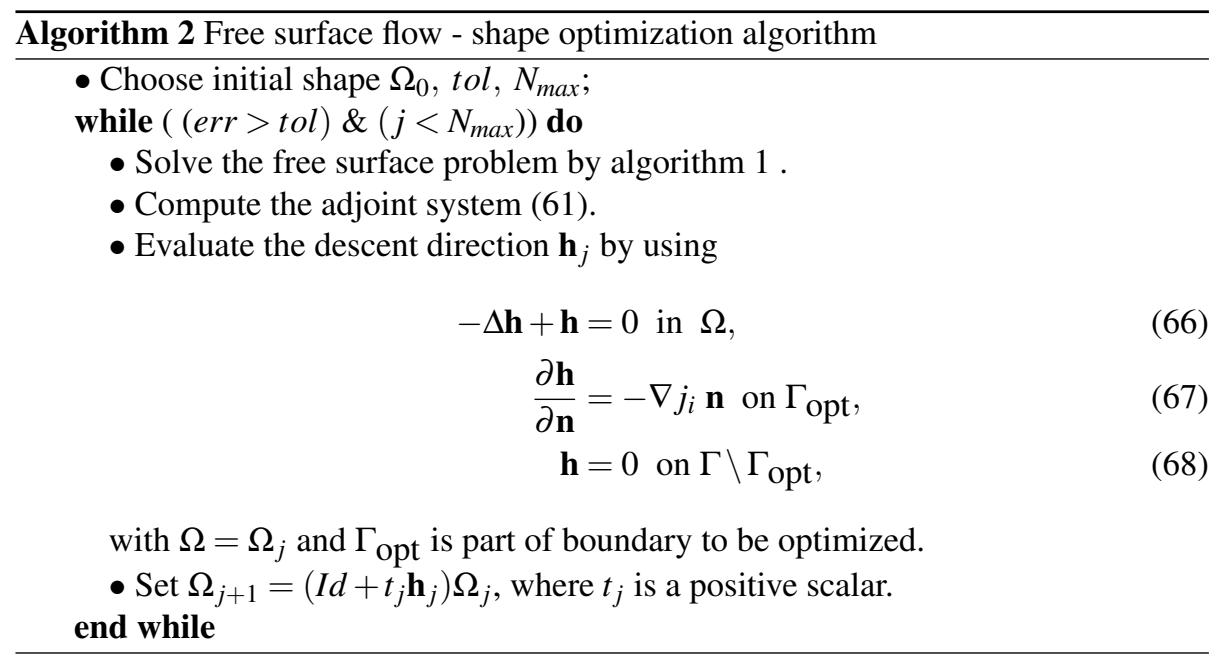

\subsection{Optimization of inflow}

In this problem, we choose parameters in $\mathscr{U}_{a d}$ as follows, $[a, b]=[-0.4,-0.2], \alpha_{\max }=$ 0.65 , and $\alpha_{\min }=0.45$, (see Figure 3). The geometry, together with the corresponding flow field depicted in Figure 2(c), are used to initialize Algorithm 2. The $\mathbf{H}^{1}(\Omega)$ norm of $\mathbf{h}$ together with the maximum value of $\mathbf{h}$ on $\Gamma_{\mathrm{opt}}$ are used as the stopping criteria for the optimization Algorithm 2, i.e., the algorithm is stopped as soon as $\max \left(\|\mathbf{h}\|_{\mathbf{H}^{1}},\|\mathbf{h}\|_{C\left(\Gamma_{\text {opt }}\right)}\right)$ is sufficiently small. During each optimization step, the step size $t_{j}$ is chosen on the basis of the Armijo-type line search and such that there are no reversed triangles within the mesh after the update. It is observed that we need on average only $k=2$ free surface flow solves per optimization step and this leads to the convergence of the optimization algorithm.

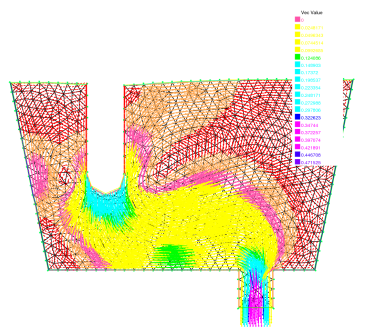

(a) $J_{1}$

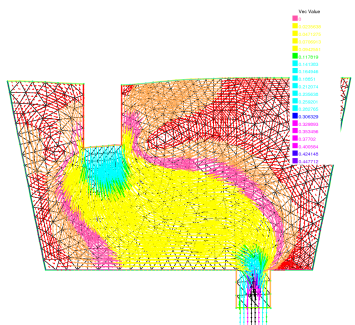

(b) $J_{2}$

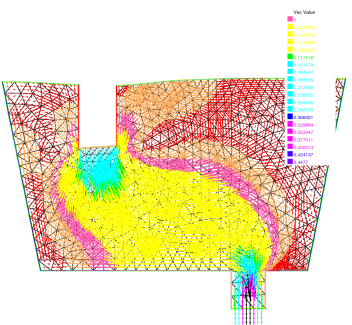

(c) $J_{3}$

Figure 4: Snapshots of optimized geometries using the three costs $J_{1}, J_{2}$ and $J_{3}$

The geometries together with the flow fields corresponding to the minimization of the three cost functionals are shown in Figure 4. The desired flow field for cost $J_{1}$ is chosen to be $\mathbf{u}_{d}=(0,-1)$, which appears to be a natural choice. Moreover, $\mathbf{u}_{d}$ is chosen to be of the same magnitude as the inflow velocity. We observe that the optimal shapes 
corresponding to the 3 cost functionals differ, with $J_{1}$ being significantly different from $J_{2}$ and $J_{3}$. For both $J_{2}$ and $J_{3}$, the depths $d$ (distance of the inflow of the bottom of the containment) increase during the optimization. For the initial and final graphs of

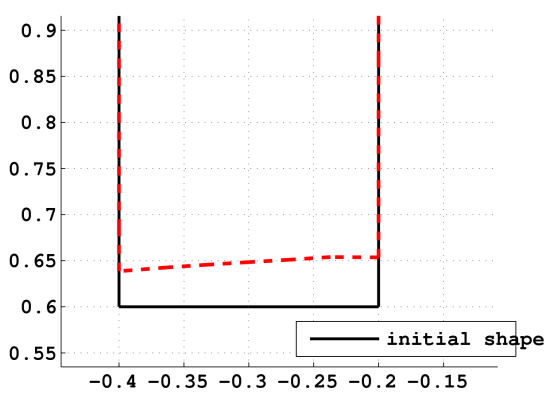

(b) $\alpha_{o p t, J_{2}}$

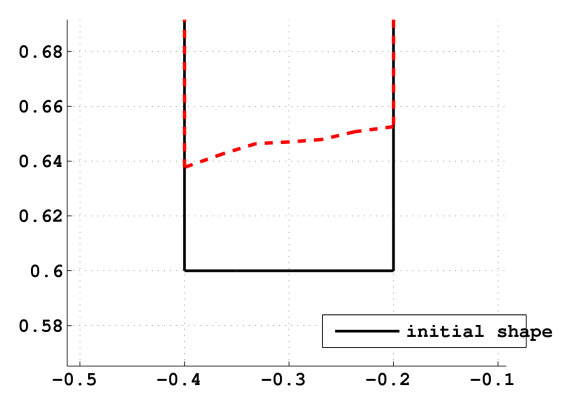

(c) $\alpha_{o p t, J_{3}}$

Figure 5: Zoom of $\alpha_{o p t}$ for $J_{2}$ and $J_{3}$

$\alpha$, see Figure 5, where the dashed line indicates the location of the optimal boundary for the inflow. The right hand end point of $\alpha_{o p t}$ is active at the upper bound $\alpha_{\max }$ for the results in Figure 5. From a physical point of view, pulling the spout out of the containment, leads to a relatively large cross section area for the net flow to occur. This results in lowering the velocities in the containment as the stream turns, and suggests a decrease of the cost functionals $J_{2}$ and $J_{3}$. We therefore compared the values for $J_{2}, J_{3}$ at $\alpha_{o p t, J_{2}}, \alpha_{o p t, J_{3}}$, to the case where all of $\Gamma_{5}$ is chosen to be active at $\alpha_{\max }$. The corresponding values are $J_{2}\left(\alpha_{o p t, J_{2}}\right)=1.011<J_{2}\left(\alpha_{\max }\right)=1.02578, J_{3}\left(\alpha_{o p t, J_{3}}\right)=$ $0.0979<J_{3}\left(\alpha_{\max }\right)=0.101275$. This suggests that a tilted spout is preferable over a straight spout. A plot of the history of the 3 cost functionals in Figure 6 clearly shows that the 3 cost functionals decrease during the minimization process.

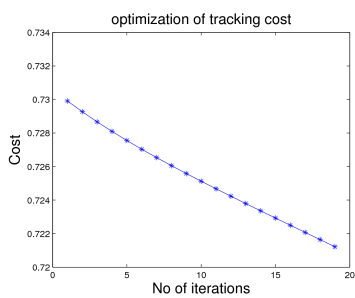

(a) $J_{1}$

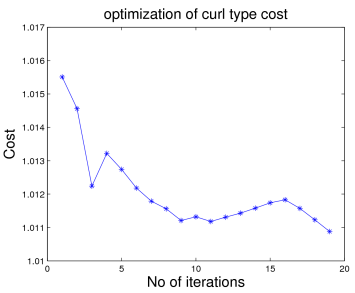

(b) $J_{2}$

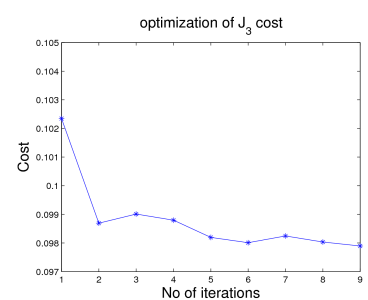

(c) $J_{3}$

Figure 6: Convergence history using the three costs $J_{1}, J_{2}$ and $J_{3}$

Remark 4.2. The optimal shape corresponding to the cost functional $J_{1}$ depends choice of the desired flow $\mathbf{u}_{d}$. A different choice would yield a different optimal shape. 


\subsection{Optimization of inflow walls}

In this example, we optimize the shape of the boundaries $\Gamma_{4}$ and $\Gamma_{6}$ of the domain $\Omega$. The parameters in $\mathscr{U}_{a d}$ (see equation (65) ) are chosen as follows, $[c, d]=[0.6,1]$, $\alpha_{\{\max , 1\}}=-0.18, \alpha_{\{\min , 1\}}=-0.22, \alpha_{\{\max , 2\}}=-0.37$, and $\alpha_{\{\min , 2\}}=0.43$. The upper ends of $\Gamma_{4}$ and $\Gamma_{6}$ are fixed at the top, i.e., $\alpha_{1}(d)=\alpha_{2}(d)=d$, while the lower ends are free. The geometry and the corresponding flow field depicted in Figure 2(c) are again used to initialize Algorithm 2. The geometries together with the flow field corresponding to the minimization of the three cost functionals are shown in Figure 7. The desired flow field for cost $J_{1}$ is again chosen as in the previous subsection. We ob-

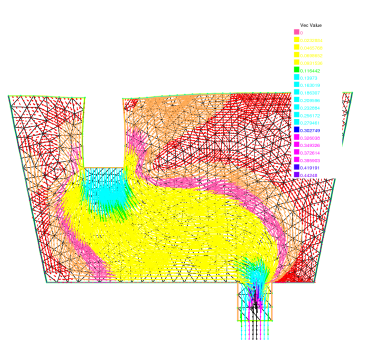

(a) $J_{1}$

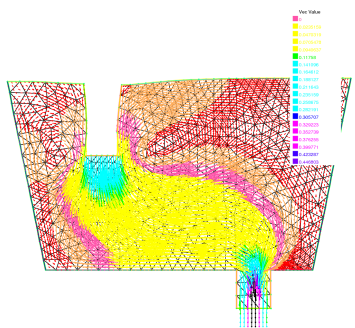

(b) $J_{2}$

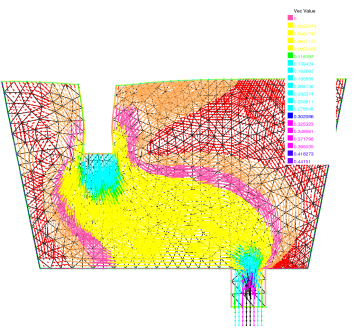

(c) $J_{3}$

Figure 7: Snapshots of optimized geometries using the three costs $J_{1}, J_{2}$ and $J_{3}$

serve that the optimal shape corresponding to the 3 cost functionals again differ, with $J_{1}$ being significantly different from $J_{2}$ and $J_{3}$. For both $J_{2}$ and $J_{3}$, their minimization leads to the narrowing of the inflow tube (see Figure 7). The converse holds for the cost functional $J_{1}$. Moreover, the plots of the history of the 3 cost functionals in Figure 8 clearly show that the 3 cost functionals decrease during the minimization process.

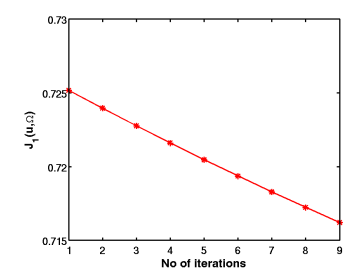

(a) $J_{1}$

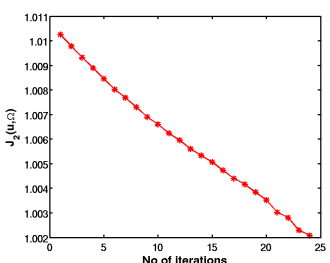

(b) $J_{2}$

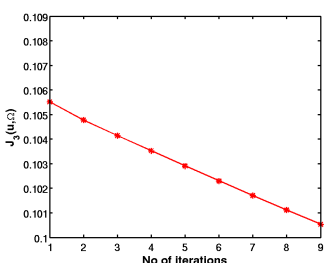

(c) $J_{3}$

Figure 8: Convergence history using the three costs $J_{1}, J_{2}$ and $J_{3}$

A physical explanation of the obtained results is the following. Momentum is transferred in a fluid by molecules in a slower-moving layer of fluid migrating to a fastermoving region of fluid and vice versa [14]. The rate of momentum transfer (I) can mathematically be expressed as

$$
I=\mathbf{u}^{2} \rho A,
$$

where $\mathbf{u}$ is the velocity of the fluid, $\rho$ the density and $A$, the cross section area of the flow region. By enlarging the inflow area $A$ while keeping the velocity $\mathbf{u}$ constant, 
the rate of momentum transfer in the fluid is increased. This consequently leads to an increase in the magnitude of the velocity field as the stream turns. The converse holds true when the inflow becomes narrower (see Figures 7 (b-c) ), which leads to a reduction in the vortex in the flow field.

\section{Conclusions}

In this paper, we proposed a new segregation algorithm for solving free surface PDE constrained shape optimization problems, by assuming that the free surface remains fixed during the optimization step. The numerical results presented indicate the feasibility of the proposed method for solving this coupled problem. It remains to investigate the relaxation of the assumption that the free surface remains fixed during the optimization and the incorporation of surface tension into the free surface model.

\section{Acknowledgment}

The second author was supported in part by the Austrian Science Fund (FWF) under grant SFB F32 (SFB "Mathematical Optimization and Applications in Biomedical Sciences").

\section{References}

[1] F. Abergel and R. Temam. On some control problems in fluid mechanics. Theoretical and Computational Fluid Dynamics, 1(6):303-325, 1990.

[2] G. Allaire and O. Pantz. Shape optimization with freefem++. Structural and Multidisciplinary Optimization, 32, 2006.

[3] S. Boisgérault and J. P. Zolésio. Shape derivative of sharp functionals governed by navier-stokes flow. In W. Jäger, J. Necăs, O. John, K. Najzar, and J stará, editors. Partial Differential Equations: Theory and Numerical Solution, pages 49-63. Chapman \& Hall/CRC Reseach Notes in Mathematics, 1993.

[4] C. Cuvelier and R. M. S. M. Schulkes. Some numerical methods for the computation of capillary free boundaries governed by the navier-stokes equations. SIAM Rev., 32(3):355-423, 1990.

[5] T. A. Davis and I. S. Duff. A combined unifrontal/multifrontal method for unsymmetric sparse matrices. ACM Trans. Math. Softw., 25(1):1-20, 1999.

[6] M. C. Delfour and J. P. Zolésio. Shapes and geometries: analysis, differential calculus, and optimization. Society for Industrial and Applied Mathematics, Philadelphia, PA, USA, 2001.

[7] D. K. Gartling and J. N. Reddy. The Finite Element Method in Heat Transfer and Fluid Dynamics. Taylor \& Francis Inc, 1994. 
[8] V. Girault and P. A. Raviart. Finite Element Methods for Navier-Stokes. SpringerVerlag, Berlin, 1986.

[9] J. Haslinger and R. A. E. Mäkinen. Introduction to Shape Optimization: Theory, Approximation, and Computation. Society for Industrial and Applied Mathematics, Philadelphia, PA, USA, 2003.

[10] F. Hecht. Bamg: Bidimensional anisotropic mesh generator. http://www.rocq. inria.fr/gamma/cdrom/www/bamg/eng.html, 1998.

[11] J. G. Heywood, R. Rannacher, and S. Turek. Artificial boundraies and flux and pressure conditions for the incompressible navier-stokes equations. International Journal for Numerical Methods in Fluids, 22(5):325-352, 1996.

[12] M. Hintermüller, K. Kunisch, Y. Spasov, and S. Volkwein. Dynamical systems based optimal control of incompressible fluids. Int. J. Numer. Methods in Fluids, 4:345-359, 2004.

[13] H. Kasumba and K. Kunisch. Vortex control in channel flows using translation invariant cost functionals. RICAM report, 08, 2011.

[14] D. P. Kesslerand and R. A. Greenkorn. Momentum, Heat, and Mass Transfer Fundamentals. Marcel Dekker, Inc, 1999.

[15] Alex G. Lee, Eric S. G. Shaqfeh, and Bamin Khomami. A study of viscoelastic free surface flows by the finite element method: Hele-shaw and slot coating flows. Journal of Non-Newtonian Fluid Mechanics, 108(1-3):327 - 362, 2002.

[16] Anastasios Liakos. Discretization of the navier-stokes equations with slip boundary condition. Num. Meth. Part. Diff. Equ, 17:200-1, 2000.

[17] P. Monk. Finite Element Methods for Maxwell's Equations. Oxford University Press, 2003.

[18] M. Moubachir and J.P. Zolésio. Moving Shape Analysis : Applications to Fluid Structure Interactions. Chapman and Hall, USA, 2006.

[19] G. Panaras, A. Theodorakakos, and G. Berggeles. Numerical investigation of the free surface in a continuous steel casting mold model. Metallurgical and Materials Transactions B, 29:1117-1126, 1998. 10.1007/s11663-998-0081-3.

[20] O. Pironneau and B. Mohammadi. Applied Shape optimization in Fluids. Oxford University Press Inc, Newyork, 2001.

[21] J. Sokolowski and J. P. Zolésio. Introduction to Shape Optimization. Shape Sensitivity Analysis. Springer-Verlag, 1992.

[22] J. I. Toivanen, J. Haslinger, and R. A. E. Mäkinen. Shape optimization of systems governed by bernoulli free boundary problems. Computer Methods in Applied Mechanics and Engineering, 197(15):3803-3815, 2008. 
[23] E. P. VanLohuizen, P. J. Slikkerveer, and S. B. G. O'Brien. An implicit surface tension algorithm for picard solvers of surface-tension-dominated free and moving boundary problems. International Journal for Numerical Methods in Fluids, 22(9):851-65, 1996.

[24] O. Volkov, B. Protas, W. Liao, and D. W. Glander. Adjoint-based optimization of thermo-fluid phenomena in welding processes. J. Eng. Math, 65(3):201-220, 2009. 